\title{
Phase space determination from measured dose data for intraoperative electron radiation therapy
}

\author{
E Herranz , J L Herraiz , P Ibáñez , M Pérez-Liva , R Puebla , \\ J Cal-González , P Guerra , R Rodríguez , C Illana and \\ J M Udías
}

\begin{abstract}
A procedure to characterize beams of a medical linear accelerator for their use in Monte Carlo (MC) dose calculations for intraoperative electron radiation therapy (IOERT) is presented. The procedure relies on dose measurements in homogeneous media as input, avoiding the need for detailed simulations of the accelerator head. An iterative algorithm (EM-ML) has been employed to extract the relevant details of the phase space (PHSP) of the particles coming from the accelerator, such as energy spectra, spatial distribution and angle of emission of particles. The algorithm can use pre-computed dose volumes in water and/or air, so that the machine-specific tuning with actual data can be performed in a few minutes. To test the procedure, MC simulations of a linear accelerator with typical IOERT applicators and energies, have been performed and taken as reference. A solution PHSP derived from the dose produced by the simulated accelerator has been compared to the reference PHSP. Further, dose delivered by the simulated accelerator for setups not included in the fit of the PHSP were compared to the ones derived from the solution PHSP. The results show that it is possible to derive from dose measurements PHSP accurate for IOERT MC dose estimations.
\end{abstract}

Keywords: Monte Carlo simulations, intraoperative radiotherapy, phase space 


\section{Introduction}

Intraoperative electron radiation therapy (IOERT) is a radiotherapy technique that delivers during a surgical intervention a single high dose of radiation directly to the tumor bed. The objective of IOERT is to achieve a higher dose in the target volume, while minimal exposure of surrounding tissues is granted either by displacing or shielding them with attenuation plates (Beddar et al 2006, Russo et al 2012). The retraction of the structures of the patient and the removal of tissues modify his/her geometry and make it difficult to carry out dosimetry calculations based on pre-operative images. In addition, as IOERT is an invasive technique that introduces an applicator to reach the tissues to be irradiated, the operatory area has to be adapted in order to achieve an ideal position of the remaining parts of the tumor. During surgery, surgeons select the applicator-cone dimension, its positioning, the bevel angle, and the energy of the electron, according to their medical and surgical experience and the information gathered during the procedure. Therefore it is difficult to plan the radiotherapy process beforehand (Pascau et al 2012, Lamanna et al 2012). IOERT is often performed by specialized units with well trained personnel. At Hospital Gregorio Marañón (Madrid, Spain), for instance, more than one thousand IOERT interventions have been performed over the last years (DiazGonzalez et al 2006, Kusters et al 2010, Pascau et al 2012).

The experience gained at Hospital Gregorio Marañón and the aim to overcome the difficulties for dose planning in the IOERT technique, motivated a joint effort (Pascau et al 2011, Santos et al 2011a, 2011b, Pascau et al 2012) to bring into the IOERT field doseplanning tools similar to the ones available for conventional radiotherapy. This effort led to the development of Radiance ${ }^{\circledR}$, the first treatment planning system (RTPS) specifically designed for IOERT (Pascau et al 2012). Radiance ${ }^{\circledR}$ has been developed under the leadership of the company GMV in collaboration with groups from several universities and the participation of several Spanish hospitals with IOERT activity (in Madrid: Hospital Gregorio Marañón, Hospital Ramón y Cajal, and Clínica La Luz, and further Hospital Regional de Castellón). In these hospitals, conventional linacs employed for external radiotherapy such as Elekta Precise SLI (Hospital Ramón y Cajal and Hospital Regional de Castellón) or Varian 21 EX (Clínica La Luz) are also used for IOERT. Radiance ${ }^{\circledR}$ allows for planning IOERT interventions from CT studies of a patient and the simulation of the whole IOERT workflow (Díaz-González et al 2006, Kusters et al 2010, Pascau et al 2011, Santos et al 2011a, 2011b, Pascau et al 2012, Guerra et al 2014).

To provide the IOERT technique with the same tools already available for conventional therapy, a MC dose planning procedure has to be developed for IOERT. Indeed, Monte Carlo (MC) methods are considered to be the most accurate methods for radiotherapy dose calculations in homogeneous and heterogeneous geometries (Rogers et al 1995, Ma et al 1999, Siebers et al 2000, McDermott et al 2003, Rogers 2006). All the relevant effects, such as material inhomogeneities, back-scatter and beam hardening can be modeled with clinically reasonable accuracy. While MC methods are computationally demanding, the development of modern computers makes them more practical in clinical settings, both for electron and photon dose calculations. A fast MC algorithm for dose calculation has been implemented into Radiance ${ }^{\circledR}$ (Guerra et al 2010, Guerra et al 2012, Guerra et al 2014).

$\mathrm{MC}$ dosimetry requires a realistic and reliable description of the electron and/or photon beams employed in the radiotherapy procedure (Chetty et al 2007). A phase space (PHSP) file containing the information of energy, angular and spatial distributions of the particles coming from the electron or photon beam, has to be fed into the MC algorithm. A common approach to build these PHSP files consists of carrying out realistic simulations of the accelerator head 
and other elements involved in the radiotherapy procedure such as applicators and collimators (Faddegon et al 1998, Ma and Jiang 1999, Capote et al 2006). Detailed MC modeling of linac systems and applicators has been widely reported (Ma and Jiang 1999, Hogstrom and Almond 2006, Sempau and Andreo 2006, Bush et al 2008, Janssen et al 2008, Jabbari and Hashemi-Malayeri 2009, Yepes et al 2009, Wysocka-Rabin et al 2011) and good results have been obtained with this method. However, this approach is limited to cases where detailed information on geometry and materials of the radiation delivery system is available (Ma and Jiang 1999, Janssen et al 2001) and requires a team with skills in MC simulations of linear accelerators, and with access to enough computing power. Moreover, MC methods in IOERT would require the simulation of each of the applicators and bevels (Picardi et al 2000, Björk et al 2002a, Ishkhanov et al 2004, Beddar et al 2006, Hogstrom and Almond 2006, Mihailescu et al 2006, Catalano et al 2007, Faddegon et al 2009, Iaccarino et al 2011, Wysocka-Rabin et al 2011).

Direct simulation of the accelerator head and IOERT applicators is unpractical in the context of the collaboration considered in this work, for which not only several different linac models for conventional therapy with dedicated applicators to deliver IOERT are employed, but it also has to consider several mini-linac specifically devoted to IOERT. Direct simulation of each linac considered in the project and the applicators involved would be too timeconsuming and it would require MC expertise and detailed knowledge of every accelerator involved. Although the development of new tools for MC simulation of clinical linear accelerators, such a PRIMO (Rodriguez et al 2103), can simplify this task, they still require a few hours of simulation for each applicator diameter, linac model and energy configuration. Further, detailed MC simulations may still present deviations in the doses computed, compared to measurements, due to inaccuracies in the treatment head geometry or the description of the electron beam (Antolak et al 2002, Faddegon et al 2005, Popescu and Bush 2005) and they would need to be fine tuned by hand.

In this work we propose an alternative method to obtain a PHSP at the exit of the applicator that can be applied within minutes without the need of special computing resources, MC skills, or detailed knowledge of the accelerator model. Instead, the information gathered from sets of dose measurements under simple reference conditions, and that usually has been gathered during the commissioning of the linac, is employed to determine complete PHSP (Faddegon et al 2009) able to produce accurate dose predictions. The fitting procedure we propose includes all the relevant variables of the PHSP, namely particle energy, position and angle. This is in contrast to previous works (Deng et al 2001) that have fit to data just a small subset of parameters of the PHSP, such as energy spectrum. Some other works, as for instance Janssen et al 2001, derived full PISPP for electrons from a set of dose measurement. In their work the electron beam is modeled as a sum of four components, namely a main and a second diverging beam, the applicator edge scatter and the applicator transmission. Each of the four components was described with a functional form and the method of simulated annealing was applied to determine the weight factors and parameters of these functions. In contrast, we propose to determine the PHSP based on pre-computed doses in uniform media without assumptions about a particular parametrized form of the PHSP. Our aim is that the final tuning to a particular accelerator may be performed at any medical physics service, with minimal or no knowledge about MC simulations of radiation delivering systems, and after only a few minutes of computation in a common computer.

In this work we present the feasibility study, thus we produce several test cases of PHSP, by means of detailed simulations of a linac, so that full knowledge of the PHSP that we want to reconstruct is available for the purpose of evaluation of the method. We picked on one side 
an applicator and linac combination representative of those employed in the Spanish hospitals considered in this project which use conventional accelerators in the IOERT procedures. Distinctive features of IOERT treatments delivered with conventional radiotherapy linac in the context of this project, are the need to consider several applicator diameters (from 4 to $16 \mathrm{~cm}$ ), bevel angles (from $0^{\circ}$ to $45^{\circ}$ ) and applicator lengths (typically from 90 to $110 \mathrm{~cm}$ ), in order to adapt the therapy to surgical findings. On the other side, we also include test cases with geometry closer to the situations found with IOERT dedicated devices, namely with a shorter applicator length.

With regards to the amount of data needed to feed the iterative PHSP reconstruction algorithm, we consider the data usually achieved at the radiotherapy services in the hospitals either during commissioning or regular quality controls of the IOERT procedures. These measurements include dose measured at several volumes of moderate size in water, for all combinations of applicator size, bevel angle and energy. In a smaller number of cases, dose measurements in air are also obtained. Thus, simulated measurements of the dose produced by the reference PHSP in air and water are computed and employed in the procedure to determine the solution PHSP. We validate our procedure applying it to the simulated dose measurements to obtain a solution PHSP. This solution PHSP is compared to the one of reference. Further, doses obtained with the reference PHSP and the solution PHSP under situations not included in the data employed in the fit are compared.

Once validated the PHSP reconstruction algorithm, PHSP for each of the LINAC accelerators included in the collaboration leading to Radiance ${ }^{\circledR}$ have been generated, from sets of measurements with each accelerator involved in this project, including some IOERTdedicated LINAC. The analysis of these data from real accelerators will be presented elsewhere. The result of this work has been integrated in Radiance ${ }^{\circledR}$, which is the only RTPS (Radiation Treatment Planning System) currently available for IOERT planning (Pascau et al 2012, Guerra et al 2014).

\section{Materials and methods}

\subsection{Phase space representation}

For the setups simulated in this work, cylindrical symmetry of the PHSP at the exit of right (i.e. bevel angle equal to zero) applicators (of cylindrical shape) can be assumed. Cylindrical applicators are used in essentially all the IOERT procedures performed at the hospitals considered in this project and cylindrical symmetry of the dose delivered by right angle applicators is assumed in IOERT planning in these hospitals. Cylindrical symmetry of the dose is measured to be within $2 \%$ level at the maximum of the PDD as part of the standard beam quality assurance procedures in the hospitals involved in this project. For the cases with bevel angle different from zero, a small piece of applicator with the adequate bevel angle is added to the MC simulation setup (see figure 1). The output factors of the non-right bevel angle applicators, are very well predicted by the MC simulations by this procedure (Guerra et al 2014).

Exploiting the cylindrical symmetry mentioned, the PHSP is fully defined by the distribution of particles in energy $(E)$, distance $\rho$ to the applicator axis, and two angles for the direction of emission: $\theta$, defined as the angle of the particle trajectory with the $z$ axis, and $\phi$, the out of plane angle. In figure 2 the definition of these angles is shown. In order to generate the particle trajectories from PHSP files encoded with the 4-uples previously described, a uniformly distributed angle has to be produced which, in combination with the distance $\rho$ to the applicator axis, defines the actual point in the $x y$ plane where the particle originates. The value of the $z$ position (along the applicator axis) of the point of origin of all the particles is assumed to 


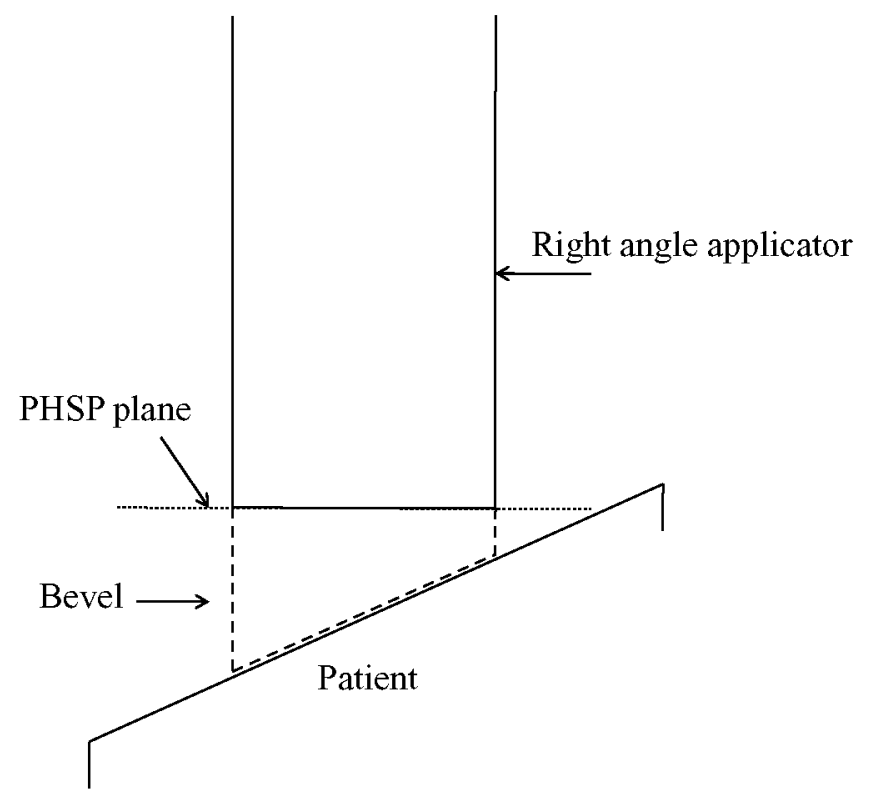

Figure 1. Schematic view of the PHSP plane at the exit of the right applicator showing the small piece of beveled applicator included in the simulation to compute the dose for non-right applicators.

be constant and it corresponds to the end of the bevel $0^{\circ}$ applicator. Particles generated by the PHSP code are represented in standard format (Capote et al 2006) by $n$-tuples which include particle type, energy, $(x, y, z)$ position and angles of emission $(\theta, \phi)$ with respect to the axis of the applicator. The decoded PHSP files can be fed into MC dose-computation packages.

To make the problem amenable to our algorithms, and in particular to allow for pre-computation of dose in homogeneous media, the $4 \mathrm{D}$ representation was used to build a discretized finite set of up to 2.000 .000 elementary sources or bins. Each bin represents a source with fixed value of energy, radial distance to the applicator axis, and trajectory angles. The number of bins chosen fits comfortably within the memory of modern personal computers. The following ranges and binning widths (BW) were considered for the definition of the elementary sources:

- Type of particle ( $\mathrm{e}^{-}$, photon).

- Energy, ranging from 0 to $16 \mathrm{MeV}(\mathrm{BW}=0.25 \mathrm{MeV})$.

- Axial angle $\theta$, ranging from $0^{\circ}$ to $29^{\circ}\left(\mathrm{BW}=0.64^{\circ}\right)$.

- Out of plane angle $\phi$, ranging from $0^{\circ}$ to $180^{\circ}\left(\mathrm{BW}=10^{\circ}\right)$.

- Radial position $\rho$ ranging from 0 to $10 \mathrm{~cm}(\mathrm{BW}=0.2 \mathrm{~cm})$.

While it is possible to use coarser bin widths in our implementation of the problem, smaller PHSP files do not speed up significantly the procedure and they actually make it more difficult for the algorithm to fit the data. The discretized version of the PHSP has some advantages. In the one hand, it maps the (infinite) number of possible solutions for the PHSP to the ones that can be represented as a combination of the bins employed here. This helps to regularize the inverse problem. Furthermore, the dose produced by each of the elementary sources (corresponding to one actual bin of the PHSP) into a given object can be computed once and stored in disk or in RAM memory, without the need to compute it during every update of the PHSP 


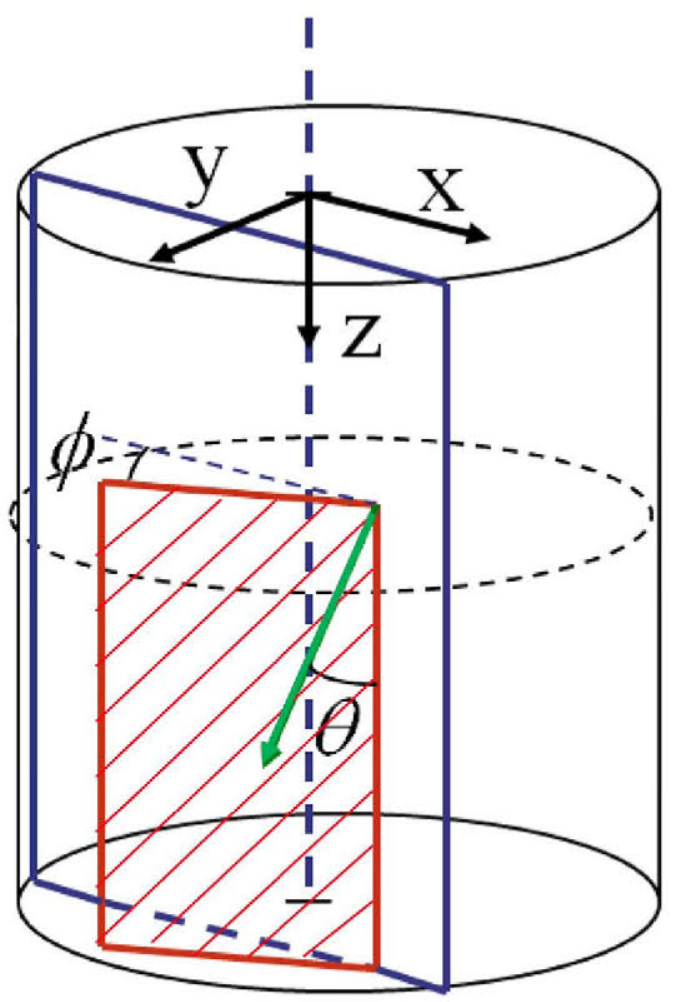

Figure 2. Angular variables employed to define the direction of a particle with the encoding of the PHSP employed in this work. The green arrow represents the trajectory of the particle, contained in the red plane (striped plane). The angle with the $z$-axis and the angle of the striped plane with the reference plane ( $x z$ plane) fully define the direction of the particle.

reconstruction algorithm. This yields an enormous reduction in the numerical computations needed to fit the PHSP to dose data in standard materials. Binned radiotherapy sources have been employed in the past (Bush et al 2008). However, it should be noticed that the geometry of the elementary sources introduced here, explicitly exhibits the cylindrical symmetry of the problem.

In figure 3 , the distributions with respect to the individual variables for the reference PHSP of the test case (to be described later) is shown. The distributions in energy, $\rho$ and $\theta$ for PHSP suitable to IOERT have been studied previously (Björk et al 2002a, Iaccarino et al 2011). However, the angle $\phi$ has received very scarce attention. We just emphasize that the dose produced by a given PHSP does depend on the distribution in this angle $\phi$, although somewhat more smoothly than on the $\theta$ angle. From symmetry considerations, the distribution in this angle $f(\phi)$ verifies $f(\phi)=f\left(360^{\circ}-\phi\right)$ and thus, it is symmetrical around $\phi=180^{\circ}$. As a general rule, $\phi$ distributions peaked at values smaller than $90^{\circ}$ would correspond to diverging beams while distributions peaked at values larger than $90^{\circ}$ would represent convergent or focused beam. Radiotherapy beams in IOERT would be mostly diverging ones, with some non-dominant converging component coming from secondary particles generated in the walls of the applicators. 
(a)

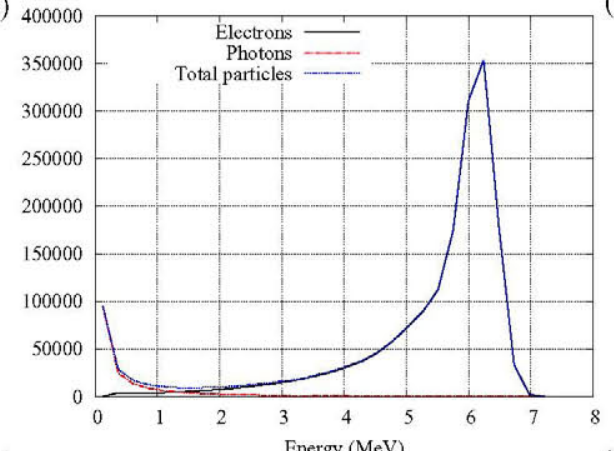

(c)

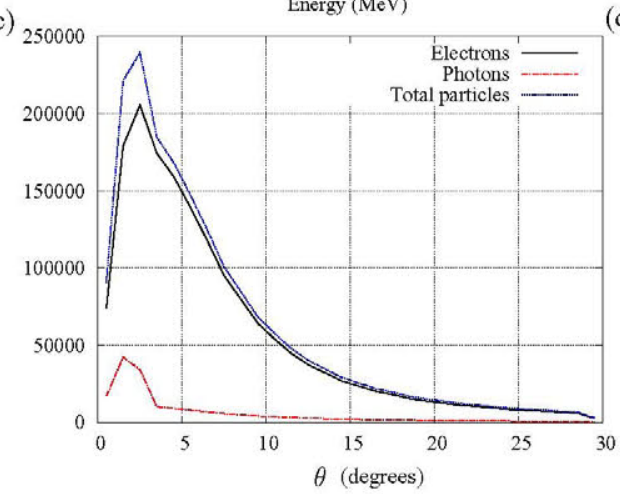

(b) 50000

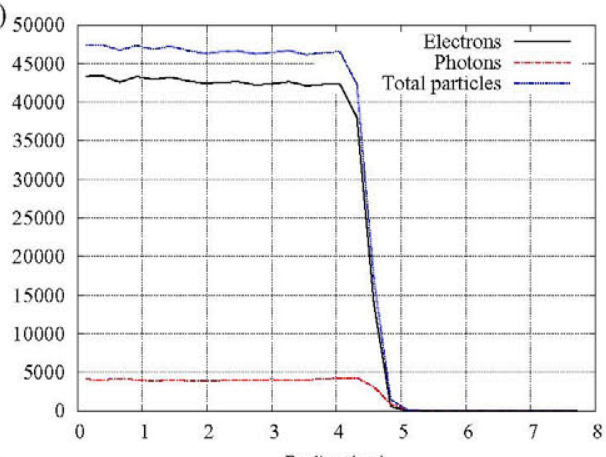

(d) 160000

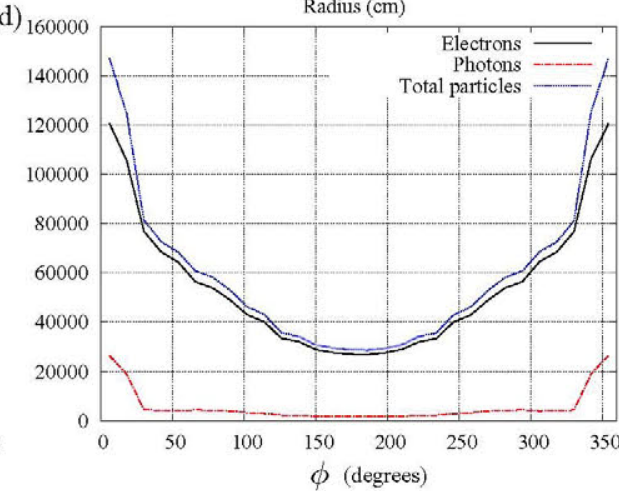

Figure 3. Histograms (normalized to 2 million particles) for the $6 \mathrm{MeV}$ reference PHSP, generated from the geometry in figure 4 , applicator length of $100 \mathrm{~cm}$, showing both electron and photon distributions. The ordinate axis shows the number of particles in each bin. The radial distribution shows the number of particles crossing the unit of surface against $\rho$, the distance to the axis of the applicator.

With regards to bin sizes, a trade-off between accuracy of the representation and number of requested bins was made. Starting from a reference PHSP made up 200 million electron particles, it was binned in successively coarser bins. The dose produced by the coarser bins was compared to the one of the unbinned PHSP. With the bin sizes employed in this work, the doses from binned and unbinned PHSP agree well within the $2 \%-2 \mathrm{~mm}$ distance to agreement gamma criteria (Bakai et al 2003, Low and Dempsey 2003, Yepes et al 2009). One has to keep in mind that once the dose is essentially indistinguishable (within any realistic measurement procedure) from the one obtained from wider bins, there is no point in employing narrower energy bins (and actually the algorithm will become unstable and would yield no useable solution), as no information remains that can help to define the energy spectrum or angular distributions with better precision.

\subsection{Simulation of the linac for the reference PHSP and doses}

In order to evaluate the method proposed, simulated data corresponding to a realistic simulation of a generic linac have been used in this work. The simulations provided not only the dose maps but also the actual PHSP, making it possible to compare directly the solution PHSP to the reference one. We will describe here the simulation of a complete accelerator treatment head for IOERT used as reference, based on the features reported by several authors (Sempau 


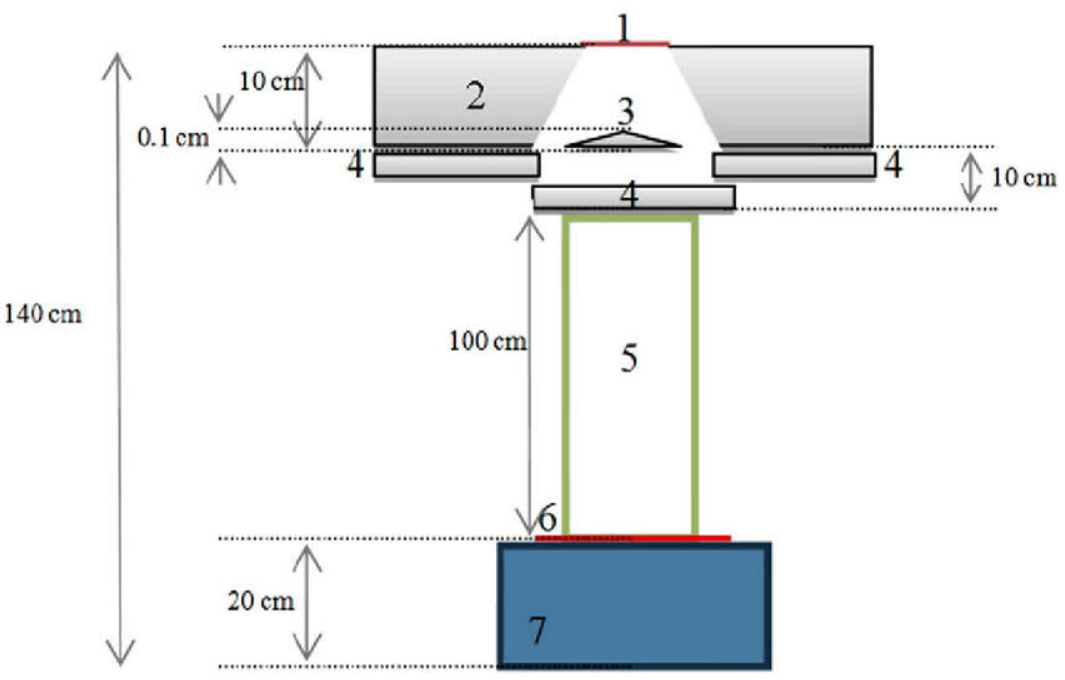

Figure 4. Schematic overview (not to scale) of the electron beam radiotherapy treatment head and electron cone setup employed for the simulations of the reference PHSP.

et al 2001, Beddar et al 2006, Mihailescu et al 2006, Wysocka-Rabin et al 2011) about the main components of the head of a linac. We included several elements specific to accelerators employed for IOERT.

The available linacs in the Spanish hospitals involved in the project are conventional linacs, mostly employed for external radiotherapy. When a conventional, non-dedicated accelerator is employed for IOERT, a specially designed IOERT applicator system must be used (Nevelsky et al 2010). These applicators are usually telescopic devices which allow changing of sourceto-surface distance (SSD) in the range of typically of 90 up to $110 \mathrm{~cm}$. The applicators considered in this work included different bevel angles $\left(0^{\circ}, 30^{\circ}, 45^{\circ}\right)$ and diameters $(3,4,5,6,7$, $8,9,10,12$ and $15 \mathrm{~cm}$ ). Although the energy range for IOERT with these conventional linacs may vary from 4 to $18 \mathrm{MeV}$, more than $95 \%$ of IOERT treatments, including all treatments in conventional IOERT settings such as breast, performed by the Spanish hospitals considered in this project, employed beam energies in between 6 and 12MeV. In the case of IOERT dedicated accelerators (Righi et al 2013), a fixed value of SSD around $60 \mathrm{~cm}$ and energies in the 4 to $12 \mathrm{MeV}$ range are typical. Therefore, in this work we have simulated two different applicators settings: one of a length of $100 \mathrm{~cm}$, representative of the average length of the telescopic applicators used in IOERT when conventional linac systems are employed (Nevelsky et al 2010) and another one of $60 \mathrm{~cm}$, typical for IOERT dedicated linacs. Surely one may think of many variations of accelerator head materials and geometries, but for most reasonable linac geometries, the conclusion on the viability of the PHSP reconstruction algorithm should be very similar to the one deduced with the geometries considered here. Beam energies of 6 and $12 \mathrm{MeV}$ were considered for the test cases, as they cover the range of energies of interest for IOERT in this project.

The linac model includes a source, exit window, primary collimator, flattering filter, secondary collimator and applicator (Wysocka-Rabin et al 2011). The schematic view of the model constructed can be seen in figure 4 .

The elements in figure 4 are as follows: 
(a) Electron window: a thin rectangular titanium foil with a thickness of $0.0055 \mathrm{~cm}$.

(b) Primary collimator: a cylinder of aluminum with a conical void shaped inner opening.

(c) Flattering filter: a conical structure of aluminum of $0.1 \mathrm{~cm}$ of height.

(d) Secondary collimator: in the case of the dedicated accelerator, this is a cylindrical collimator made of $2 \mathrm{~cm}$ thick aluminum and an opening for the PMMA applicator of the adequate diameter to insert the applicator. In the case of conventional accelerator, it is composed of the usual rectangular collimator, representing fully open multileaf collimator jaws, followed by a $3 \mathrm{~mm}$ thick steel collimator, with a cylindrical opening adequate to insert the PMMA applicator.

(e) Applicator: a PMMA cone with cylindrical walls of thickness $0.5 \mathrm{~cm}$, inner radius of $4.5 \mathrm{~cm}$. As we described above, two lengths $(60$ and $100 \mathrm{~cm})$ have been simulated. The geometries of the secondary collimators and applicators, in particular wall thickness, have been chosen to mimic the setups found in the actual treatments by the hospitals involved in this project.

(f) Scoring plane: A rectangular region which sets out the trajectories of the particles that cross it.

(g) Water or air phantoms: a block of water/air with dimension $20 \times 20 \times 20 \mathrm{~cm} 3$.

The simulations were performed with the Monte Carlo package penEasy based on PENELOPE (Ma and Jiang 1999, Sempau et al 2001, Sempau et al 2003, Faddegon et al 2009). This MC package is fast, easy to use and very accurate, and it has been extensively benchmarked.

Electron sources with a Gaussian energy distribution with a mean energy of $6 \mathrm{MeV}$ and $12 \mathrm{MeV}$ have been simulated. In both cases, with a FWHM of $0.5 \mathrm{MeV}$. With respect to the spatial distribution of the source, it was taken a Gaussian distribution centered at zero with a full-width-half-maximum (FWHM) of $0.1 \mathrm{~cm}$. The sources were simulated as circular foils of $0.5 \mathrm{~cm}$ radius placed $0.5 \mathrm{~cm}$ above the electron window. It has to be noticed, however, that for IOERT accelerators, the PHSP at the end of the applicator does not depend much on small details of the source of the electrons at the top of the accelerator head (Björk et al 2002b). We have verified that the PHSP at the scoring plane of the simulation fulfill the cylindrical symmetry at the level of the statistical uncertainty of the simulation (around 2\%). For the actual accelerators, dose uniformity better than $2 \%$ in water phantoms inside circle areas perpendicular to the beam direction are verified.

Enough initial electron particles were generated in order to accumulate more than 200 million particles in the scoring plane. The simulation took around $50 \mathrm{~h}$ on a single core of an Intel(R) Xeon(R) E5620 at $2.40 \mathrm{GHz}$, for each energy and applicator. The corresponding PHSP obtained at the exit of the applicator is taken as our reference problem. Histograms for each of the four relevant variables can be seen in figure 3. For simplicity, we discuss at length the case of $6 \mathrm{MeV}$ energy and applicator length of $100 \mathrm{~cm}$. The other cases studied are more summarily presented in section 3 .

\subsection{Description of the algorithm to determine the PHSP from dose measurements}

The optimization procedure to derive a PHSP from a set of dose measurements is based on an implementation of a Maximum Likelihood Expectation Maximization (EM-ML) iterative algorithm, commonly used for inverse problems like image reconstruction (Herraiz et al 2006). The estimated dose would be given by $D_{j}^{\text {th }}=\sum_{i} w_{i} d_{i j}$, where the relative weights $w_{i}$ of each elementary source are iteratively fitted in order to reproduce the experimental dose. Here, $i$ represents the index of the elementary source (a bin) of the PHSP, $j$ is the index of a given 
voxel of the volume of interest, $D_{j}^{\exp }$ is the experimental dose at voxel $j, D_{j}^{\text {th }}$ is the estimated dose at that same voxel, and $d_{i j}$ is the dose produced by source $i$ in voxel $j$. This algorithm seeks the PHSP with maximum likelihood of having produced the observed dose according to the update prescription for the weights $w_{i}$.

$$
W_{i}^{\prime}=W_{i} \frac{\sum_{j} d_{i j} \frac{D_{j}^{\text {exp }}}{D_{j}^{\text {th }}}}{\sum_{j} d_{i j}} .
$$

That is, each weight is updated by the weighted-average value of the ratios of experimental to theoretical doses. The weights represent the influence of a given bin in the dose received by a given voxel, where in this work weights are taken proportional to the dose produced by bin $i$ in voxel $j$. The iterative procedure consists of two main steps: forward and backward projection. The algorithm begins with an initial PHSP, given by a certain population of each bin with a number of particle trajectories. From this PHSP, the dose produced in the materials where the dose measurements are made, is estimated. For each bin of the PHSP, the dose $\mathrm{d}_{\mathrm{i}}$ is obtained with the DPM dose-computing code (Sempau et al 2000). By the principle of linear superposition of doses, the total dose in the volume of interest is obtained as a linear combination of the individual dose foot prints $\mathbf{d}_{i}$ deposited by each of the elementary sources. This part of the algorithm is termed forward-projection or, simply, projection, and during it the dose estimates $D_{j}^{\text {th }}=\sum_{i} w_{i} d_{i j}$, are computed.

The measured dose, $D_{j}^{\text {exp }}$, is then compared with the estimated one, $D_{j}^{\text {th }}$, and correction factors are obtained for each voxel. Then, the backward projection stage of the algorithm is performed. At this stage of the calculation, the ratio of measured to estimated dose is obtained for each voxel. These ratios are combined in a weighted average value to yield a multiplicative correction factor for each bin.

The above mentioned doses $d_{i j}$ for each bin can be pre-computed using a cluster and stored in a set of $(\rho, z)_{i}$ maps. The use of pre-computed dose speeds up projection and backward projection steps. For instance, for the cases considered here, and even though DPM is a very fast code, the total evaluation of the doses $d_{i}$ with 1 million particles per bin, for all the bins required in this work, would take the equivalent to 10 -months of a single-core processor. A full iteration is completed when the multiplicative factor has been computed for all bins and the PHSP is updated applying these multiplicative factors to each bin. Then, a new iteration begins with the updated PHSP. The procedure stops when convergence is reached, which typically requires around 200 full iterations. Using pre-computed doses, these 200 iterations take a few minutes in a single-core of the processor mentioned above.

\subsection{Dose measurements used in the fiting algorithm}

Besides the initial PHSP, measurements in homogeneous media are the other ingredient of the fitting algorithm. For these measurements, different data sets were simulated and employed in the fitting procedure. In one side, solution PHSPs obtained employing only data in water are obtained. In this case, dose in an homogeneous body of water at the exit of the applicator for $4000 \rho, z$ pairs values is assumed known, namely for $z$ from 0 to $20 \mathrm{~cm}$ and $\rho$ from 0 to $8 \mathrm{~cm}$, in steps of $0.2 \mathrm{~cm}$. Another set of solution PHSPs are obtained employing in the fitting algorithm dose from homogeneous volumes of the same size and detail in both water and air. Although this volume and detail of data poses no problem for our simulated reference problem, in a more realistic case, and indeed for the cases considered in this project, most often just the PDD and a few transverse profiles would be known. In such a case, suitable interpolation 
procedures could be employed in order to obtain the $4000 \rho, z$ pairs data to be fed into the iterative algorithm. We have verified (Herranz et al 2011, Herranz et al 2012a 2012b Ibañez 2012, Pérez-Liva 2012) that, provided a PDD profile is extracted from the known dose, with resolution of the order of $2 \mathrm{~mm}$, and transverse profiles with resolution of a few $\mathrm{mm}$ and spaced for instance every $2 \mathrm{~cm}$ in the $z$ direction are available, then a suitable interpolation procedure can be found so that the interpolated dose is within $1 \%-1 \mathrm{~mm}$ of the actual simulated dose. Resorting to this interpolation technique, the volume of data needed to apply the algorithm is comparable to what the Medical Physics Services obtain during commissioning of IOERT applicators. Feeding the iterative algorithm with the whole set of 4000 pairs of $\rho, z$ values regularizes the solutions and prevents the algorithm to consider solutions with unrealistic dose behavior outside the referenced points.

\subsection{Methods to deal with the ill-posedness of the PHSP reconstruction problem}

The reconstruction of the PHSP, as described here, is a typical ill-posed problem (Bakushinsky et al 2011) for several reasons. In one side, the number of reference data is limited. Even if the available modern dose measurement devices can record the dose in a volume of waterequivalent materials (at times also air), normally the number of data points to fit (in our case the dose at $4000(\rho, z)$ pairs) would be much smaller than the number of elementary sources that have to be determined. On the other hand, tiny variations in the weights in the PHSP may result in unnoticeable changes, within the limits of the measurement devices, on the dose recorded. This means that different PHSP solutions may be equally valid (i.e. the solution is not unique). Finally, if no regularization of the algorithm is used, possible small variations in the data (noise) may yield large variations in the final solution (possible lack of stability or robustness).

These problems can be alleviated to some extent by including complementary measurements in more than one media, reducing the number of variables, factorizing the solution, using a realistic initial PHSP estimation in the iterative algorithm, regularizing the method, and filtering the possible noise in the dose measurements. Here we describe the main steps evaluated and followed to obtain accurate and valid solutions in this ill-posed problem.

2.5.1. Increase the amount of reference data. The proposed method is not limited to the use of any specific dose measurement as an input. We have evaluated two possible scenarios: one where only dose measurements in water are present, and other in which dose in water and in air are available. These are the most common measurements that are performed in a clinical setting. Of course, if more data is available for the fitting procedure, it can be used to constrain the space of possible solutions even more, making it less sensitive to the initial PHPS distribution. As it can be seen in figure 5, dose measurements in water, due to its relatively low penetration, exhibit small sensitivity to small variations in the direction of the beam. Figure $5(c)$ shows how beamlets in water with significant different angle $\theta$ have much similar dose distribution between them than the corresponding beamlets in air (figure $5(d)$ ). This makes it difficult for our method to properly reconstruct the angular variables based only on measurements in water. On the other hand, measurements in air are specially useful for the reconstruction of the angular variables (figures $5(b)$ and $(d)$ ), although the larger penetration in air provides little information on the energy of the electrons. Therefore, a combination of these two measurements, if available, would be the best option for the proposed method. 
(a)

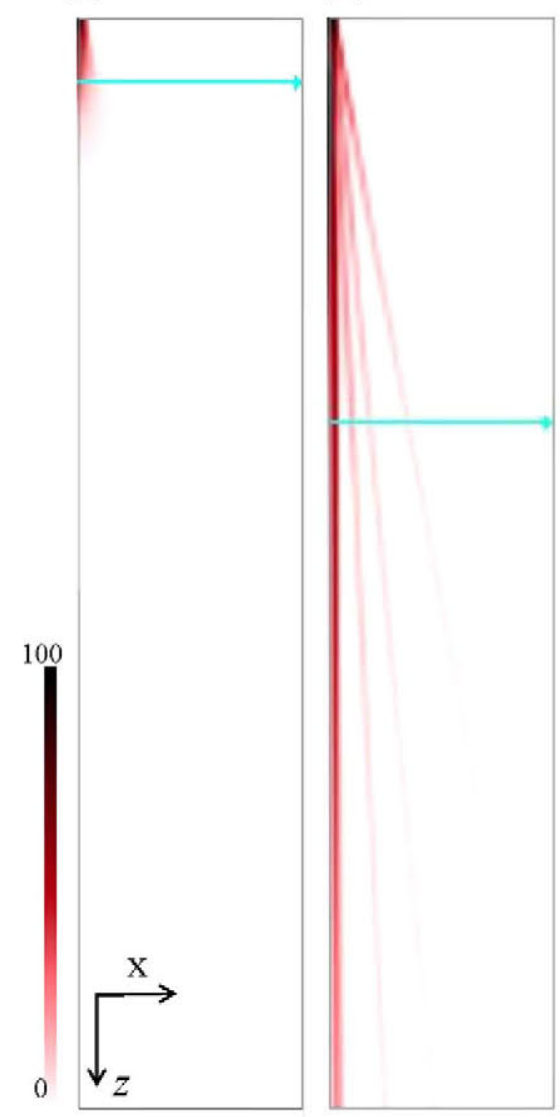

(c)

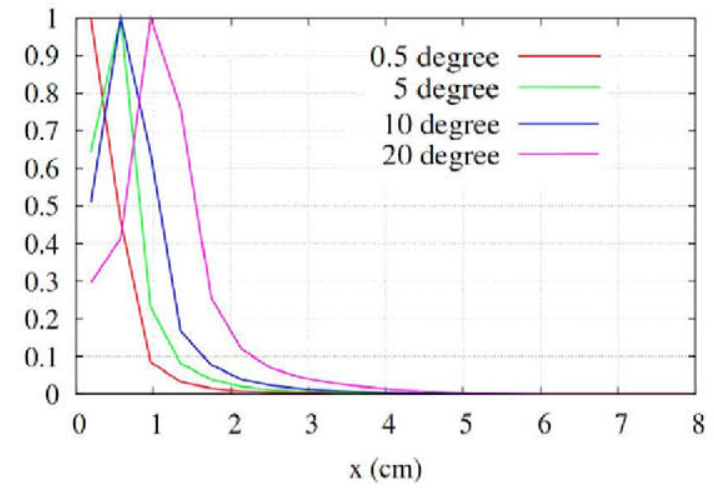

(d)

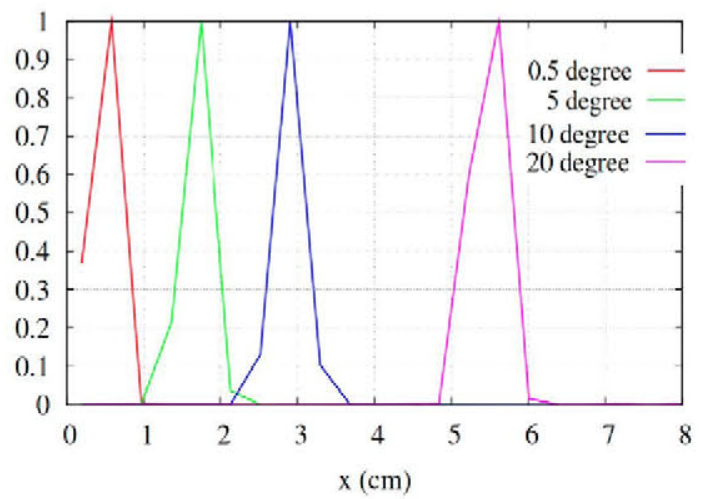

Figure 5. Beamlets of $6 \mathrm{MeV}$ for a theta angle of $0.5^{\circ}, 5^{\circ}, 10^{\circ}$ and $20^{\circ}$ degrees in $(a)$ water and $(b)$ air and dose profile along $x$-axis corresponding to these beamlets in water (c) and air $(d)$

2.5.2. Reduction of variables. In this work, photons are not treated independently in the fit due to their low contribution to the dose. In IOERT, the production of photons should be relatively small. The dose obtained from both electrons and photons can be compared to the one resulting only from the electrons. It can be seen that, where the dose is significant, the photons contribute very little (less than $3 \%$ at the maximum of the PDD) to the dose. This means that it would be extremely difficult to obtain a sensible photon distribution in the solution of our inverse problem, where the data are greatly dominated by the electron contribution. We have chosen to look for a solution PHSP that completely lacks photons. This is not a strong simplification, as the bulk amount of dose in the region of interest in IOERT comes from the electrons, being the photons a very small fraction and, further, the role of photons will be replaced in the solution PHSP, at least to some extent, by electrons of low energy. In those cases where the photon contribution to the dose may be significant, it can be determined with the methods described in the literature (Faddegon et al 2000, Chvetsov and Sandison 2002).

2.5.3. Factorization of the algorithm. A distinctive characteristic of our approach is that the PHSP can be obtained minimizing simultaneously on the four $\rho, E, \theta$ and $\phi$ variables. 


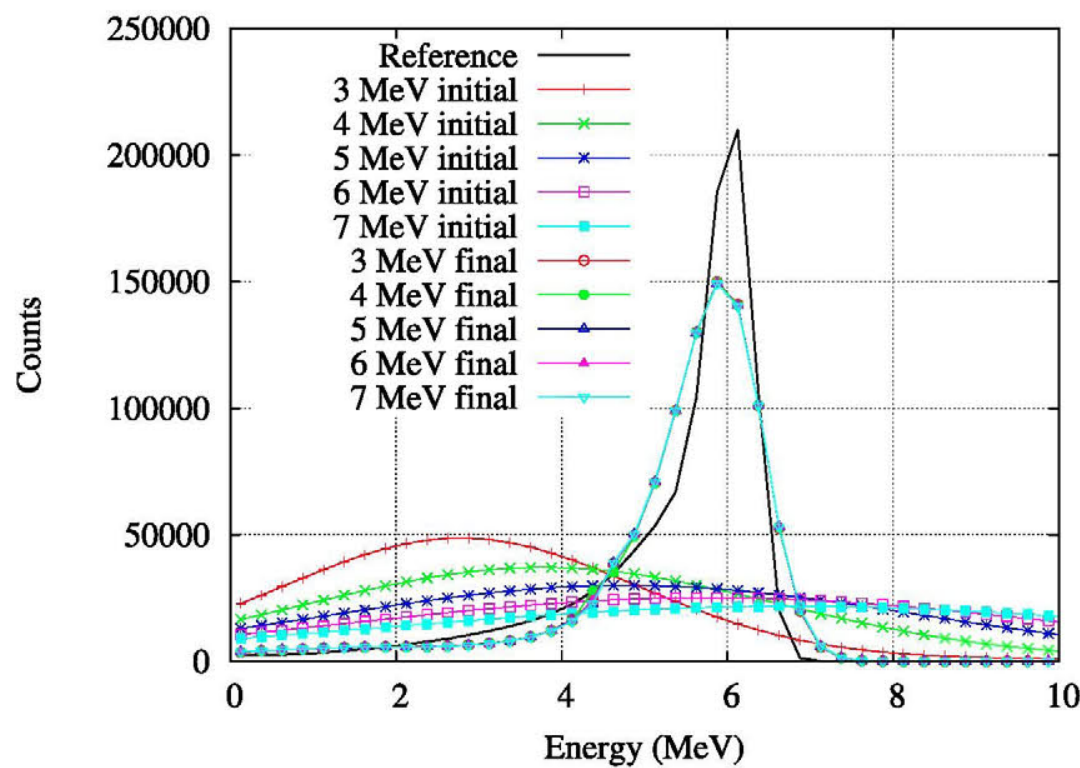

Figure 6. Example of energy distribution of particles obtained from different initial energy distributions centered at different values of the energy, for the case of $6 \mathrm{MeV}$ PHSP. We can see that the final energy distribution is rather independent on the initial distribution employed. The energy distribution of the reference PHSP is also shown.

Solutions can be also obtained by minimizing only one of them each time, instead of all variables at the same time. Nevertheless, although it could seem to be a good way to simplify the PHSP reconstruction, we observed that in this latter case, convergence is somewhat slower and the optimization procedure tends to be caught more often in sub-optimal local minima. Therefore, in this work, we have only used this approach in one test case, in which we fit the energy distribution while keeping the rest of the variables with their initial distributions (see cases 5 and 6 described in next section).

2.5.4. Use of a realistic initial PHSP. The use of a good initial guess of the solution is crucial in many ill-posed problems (Bakushinsky et al 2011). In this section we explain how the initial estimations of the distribution in each variable were selected. It is important to note that as the algorithm works by updating with multiplicative factors the weight of the bins (equation (1)), if a bin ever gets a zero weight, that bin will not longer play a role in the optimization. Therefore, initial PHSP estimations should not have any zero weights, warranting that every bin could contribute to the dose.

- Radial distribution $(\rho)$ : the initial distribution of particles in the radial direction was taken proportional to dose profiles right after the applicator, that is, in the first couple of $\mathrm{mm}$ of object, plus a small background to avoid zero weights. This is a reasonably starting guess for any accelerator configuration.

- Energy distribution $(E)$ : with regards to the initial energy distribution, we use a very broad Gaussian distribution centered around $5 \mathrm{MeV}$ and with a FWHM of $5 \mathrm{MeV}$, for the case of $6 \mathrm{MeV}$ PHSP, and centered around $11 \mathrm{MeV}$ with a FWHM of $11 \mathrm{MeV}$, for the $12 \mathrm{MeV}$ PHSP. This procedure is a reasonable approximation to an arbitrary initial energy distribution, also provides non-zero weights in the region of interest and indeed we have verified that the converged energy distribution is rather independent on the initial energy distribution employed (see figure 6). 
Table 1. Cases evaluated for the different options in the initial angular distribution, the amount of measurements used and the number of variables adjusted.

\begin{tabular}{|c|c|c|c|c|c|c|c|c|}
\hline \multirow[b]{2}{*}{ CASE } & \multicolumn{2}{|c|}{$\begin{array}{l}\text { Initial angular } \\
\text { distribution }\end{array}$} & \multicolumn{2}{|c|}{$\begin{array}{c}\text { Dose } \\
\text { measurements } \\
\text { used as input }\end{array}$} & \multicolumn{2}{|c|}{ Variables adjusted } & \multicolumn{2}{|c|}{$\begin{array}{c}\text { Nominal } \\
\text { energy } \\
(\mathrm{MeV})\end{array}$} \\
\hline & Analytical & Flat & Water & Air & $\rho, E, \theta, \phi$ & $E$ only & 6 & 12 \\
\hline 1 & $x$ & & $x$ & $x$ & $x$ & & $x$ & \\
\hline 2 & & $x$ & $x$ & $x$ & $x$ & & $x$ & \\
\hline 3 & $x$ & & $x$ & & $x$ & & $x$ & $x$ \\
\hline 4 & & $x$ & $x$ & & $x$ & & $x$ & \\
\hline 5 & $x$ & & $x$ & & & $x$ & $x$ & \\
\hline 6 & & $x$ & $x$ & & & $x$ & $x$ & \\
\hline 7 & $70 \%$ & $30 \%$ & $x$ & $x$ & $x$ & & & $x$ \\
\hline
\end{tabular}

- Angular distribution ( $\theta$ and $\phi$ ): for the initial angular distributions, we considered two extreme approaches. In the first one (i) a flat (uniform) distribution on both angles is employed as initial distribution. This is a not very realistic initial guess for the fitting procedure but it certainly does not contain any information on the problem to solve and also warrants non-zero weights in the starting PHSP. In the other extreme, (ii) angular distributions given by analytical relationships presented in Jannsen 2001 (Janssen et al 2001) are taken, assuming only primary electrons in the initial distribution. This second choice may have the problem of not fully populating all angular bins. Thus, combinations of a PHSP with an angular distribution based on only primary electrons plus another one with flat angular distributions have also been tested (see case 7 in table 1).

2.5.5. Regularization of the algorithm. One of the main characteristics of the PHSP is that the distribution in the four variables considered is smooth (see figure 3 ). We used this fact to regularize the algorithm by starting with a smooth initial PHSP estimation and stopping the iterative algorithm before it starts overfitting the data and the noise. This is a typical method used in the EM-ML algorithm (Herraiz et al 2006). Other regularization methods are possible, like one-step late maximum a posteriori (Green 1990), although we did not use them in this work.

2.5.6. Data noise reduction. As explained in section 2.4, when dealing with real data, a fitting procedure will be used to create a uniform sampling of the dose over the volume of interest. This fitting procedure is based on the assumption that dose distributions are smooth, similarly to what happens with PHSP distributions. Therefore, we can fit the dose to smooth functions, eliminating most of the noise that could be present in the measurements and affect the reconstructed PHSP. Further, the cylindrical symmetry assumption reduces the noise in the data.

To illustrate the convergence of the method and how possible noise in the data propagates into the PHSP solution we show in figure 7 the evolution of the energy and $\rho$ distribution for different number of iterations (1,50 and 100 iterations) using dose simulated measurements with (a) low noise (simulated data with 200 millions of histories) and (b) significant noise (simulated data with 20 millions of histories). 

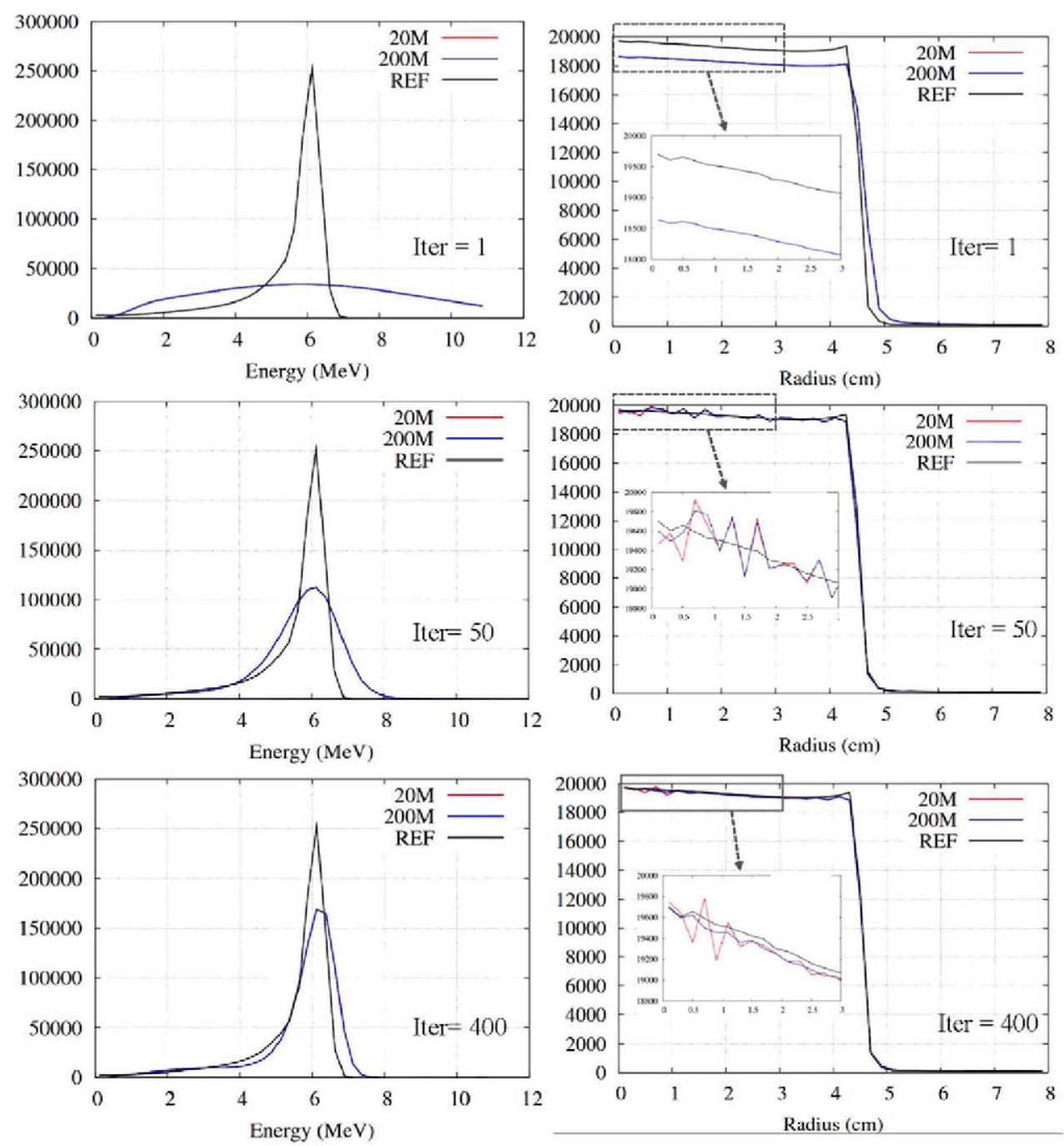

Figure 7. Convergence of the algorithm in the energy (left) and $\rho$ (right) distribution for two cases: $(a)$ low noise (200M histories), and $(b)$ significant noise (20M histories) for different number of iterations $(1,50$ and 100$)$.

In this work, the proposed algorithm has been tested using different combinations of initial PHSP distributions, different amount of input data and variables to test to which extent the procedure is capable of reaching a reasonable PHSP (table 1).

Although not shown in this paper for the sake of clarity and brevity, case 7 was also introduced for the $6 \mathrm{MeV}$ energy, and cases 1 to 6 for the $12 \mathrm{MeV}$ energy. The findings in these cases are similar to the ones for the cases shown here.

\subsection{Additional setups to validate the dose obtained from the solution PHSP}

We fit the PHSP from dose volumes in water and air, and the resulting PHSP can be compared to the reference one. However, in a general case the deviations of the solution PHSP from 
the reference one would only be assessed in terms of difference in the dose they produce, for representative cases that have different content from the one employed in the fitting of the solution PHSP. Thus, we have prepared several additional setups, besides the right angled applicator and homogeneous media, and we have compared the dose obtained with the reference PHSP and the one obtained with each of the solution PHSP described in the previous subsection. The additional setups correspond to:

2.6.1. Applicator. Increasing the applicator length. We take the PHSP determined from our measurements and propagate it along $10 \mathrm{~cm}$ of additional applicator. We compare the doses in a homogeneous water phantom obtained this way with the ones of the reference PHSP. This is an important test showing the predictive power of the solution PHSP to estimate the variation of the output factors with the applicator length, and thus with the SSD. Due to the nature of the IOERT procedures to which this work is oriented, when using conventional applicators, it is often needed to adapt the length of the applicator to match the conditions of irradiation after surgery. $A \pm 10 \mathrm{~cm}$ variation on the SSD around the reference value is typical. This test is very sensitive to the amount of divergence of the beam, and thus the solution PHSP must exhibit a similar angular dependence to the reference PHSP in order to pass this test.

2.6.2. Bevel. Applicator end with a bevel angled at $45^{\circ}$. The solution PHSP is introduced into a short length of applicator ended at $45^{\circ}$ and applied to a homogeneous water phantom. We compare the dose delivered to the water phantom with the solution PHSP to the one of the reference PHSP for the same setup. Again, beveled applicators are very often used in IOERT (Björk et al 2000, Nevelsky et al 2010, Russo et al 2012, Righi et al 2013) and in particular in near half the clinical cases considered for this project. $45^{\circ}$ is typically the maximum bevel angle employed.

2.6.3. Lung and bone. Non-homogeneous phantom. In real situations, the treatment volume would include non-homogeneous tissues. In these tests, a geometry including water and a lower (lung, $0.26 \mathrm{~g} \mathrm{~cm}^{-3}$ (ICRU 44, 1989)) or larger (bone) density tissues are studied. As in the previous cases, the doses obtained with solution and reference PHSP are compared. This lung, tissue (water) and bone setup resembles breast IOERT procedures, one of the most prevailing IOERT scenarios.

2.6.4. Lead. Use of shielding disks. One of the objectives during IOERT treatment is the shielding of healthy tissue from radiation (Catalano et al 2007, Russo et al 2012). The surgeon places a metal disk to stop the electron beam and to protect the tissues located beyond the disk In this test it is simulated a lead shield inside a water volume.

\subsubsection{Step. Phantom with both air and water data.}

These test cases are depicted in figure 8 .

For all these setups we compare the doses produced in terms of the gamma evaluation (Bakai et al 2003, Low and Dempsey 2003, Yepes et al 2009). The dose obtained with the true PHSP from the simulated linac and the ones obtained from reconstructed PHSPs are compared for the homogeneous water or air phantoms, as well as for the inhomogeneous setups. The comparison was performed with $1 \%-1 \mathrm{~mm}, 2 \%-2 \mathrm{~mm}$ and $3 \%-3 \mathrm{~mm}$ criteria (Alber et al 2008). We would say that a given solution PHSP passes a given gamma criteria (say $1 \%-1 \mathrm{~mm}$ ) if at least $95 \%$ of the voxels with dose equal or larger than $5 \%$ of the maximum dose present a gamma value smaller than one. The results are quoted in terms of the most demanding gamma criteria, of the three above mentioned, that a given case passes. If a case 

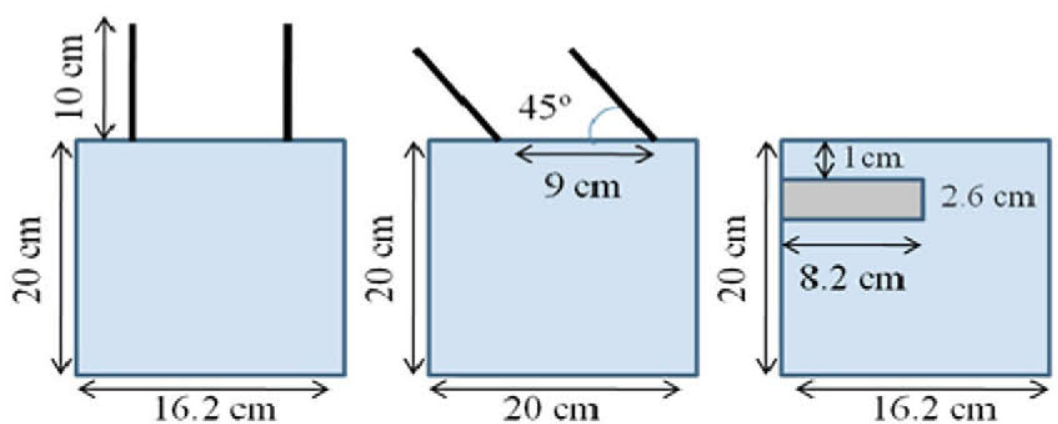

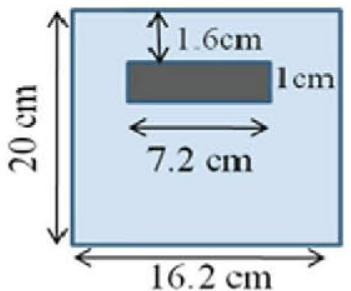

Lead

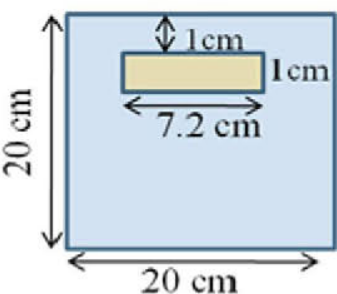

Bone

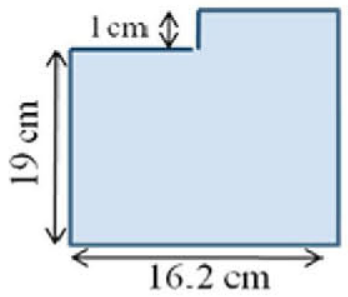

Step

Figure 8. Validation setups where the dose produced by solution and reference PHSP are computed and compared. The PHSP are applied at the upper end of the setups and the dose is computed in the water or water + lung, lead or bone objects.

does not pass even the $3 \%-3 \mathrm{~mm}$ gamma criteria, then the percentage of voxels with gamma smaller than one for the $3 \%-3 \mathrm{~mm}$ is presented.

\section{Results and discussion}

\subsection{Convergence and comparison with the reference PHSP}

In this section, we show a comparison of the PHSP obtained under different conditions for the electron source of $6 \mathrm{MeV}$ (figure 9) and $12 \mathrm{MeV}$ (figure 10). It is expected that the PHSP obtained would differ to some extent from the one employed to generate the reference dose. With regard to the energy, we have seen already that the energy distribution of the converged PHSP solution is rather independent on the initial energy distribution. We see in both figures 9 and 10 that it is also very similar for the cases studied (in figure 9, cases 5 and 6 are not shown for clarity, but the energy distribution is also very similar to the ones of cases 3 and 4 ). These energy distributions are not identical to the reference one (also shown in figures only for electrons in the reference PHSP), mainly because their FWHM is somewhat larger, but the centroid and general shape of the energy distribution agrees well with the one of the reference PHSP. The larger FWHM can be attributed in one side to the finite bin size in energy employed. Actually, the resulting energy distribution of the solution PHSP is approximately what one can expect if the original energy distribution is blurred with the energy bin size employed, in this case $0.25 \mathrm{MeV}$. As explained in section 2, it is only possible to distinguish results of narrower bins when these narrower bins can produce a measurable effect in the dose. 

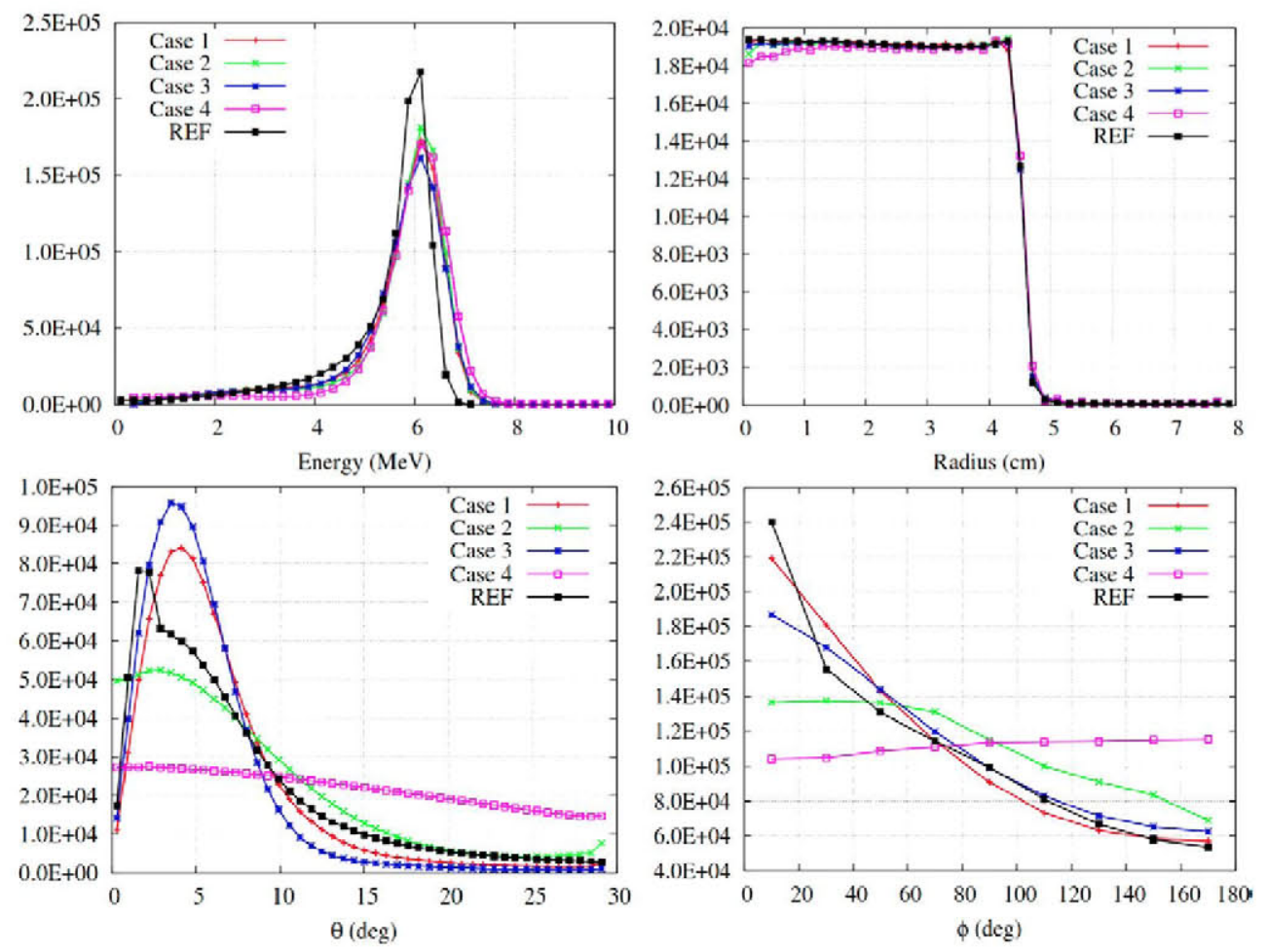

Figure 9. Distribution of electron particles for the solution PHSP for cases 1 to 4 , for the $6 \mathrm{MeV}$ reference PHSP and $100 \mathrm{~cm}$ applicator length. In all cases, the histograms are normalized to the same total number of particles. It can be seen that distributions in both energy and distance to the applicator axis, the four solutions are similar and very close to the reference PHSP. For the angular distributions, however, differences are visible. Actually, for case 4 , the angular distribution does not show a visible peak in $\theta$ and in $\phi$ it is peaked to values larger than $90^{\circ}$, featuring then a converging beam distribution.

Within this limitation, we can conclude that the energy distribution of the reference PHSP can be well recovered by solving the inverse problem, even if only data in water are employed.

With regard to the $\theta$ distribution, looking at figure 9 , it stems out is that it is very difficult to obtain proper angular dependences in the reconstructed PHSP if only data in water are employed and a flat angular initial distribution is used as an input in the procedure (case 3 and 4). The reason for this behavior is explained with more detail in section 3.2. It is important to note that the solution PHSP for case 2, with flat initial angular distributions, is able to recover a distribution that follows the trend of the realistic simulation. Both solution PHSP for cases 1 and 2 peak at similar values than the reference PHSP, having case 1 a smaller tail for large angles than the reference PHSP, and case 2 a somewhat larger one. For the solution PHSP of case 3 and 4, which do not use data in air, the deviations from the reference distribution are much more evident. Case 3 lacks almost completely the tail in the distribution at larger $\theta$ angles and case 4 has an almost flat distribution. Similar conclusions are derived from the $\theta$ distribution in figure 10, where case 7 can be considered a mixture of cases 1 and 2 , and obtained better results than case 3 .

With regard to the $\phi$ distribution, there are visible differences in both figures 9 and 10 between the solution distributions and the reference ones. As we will see in next sections, 

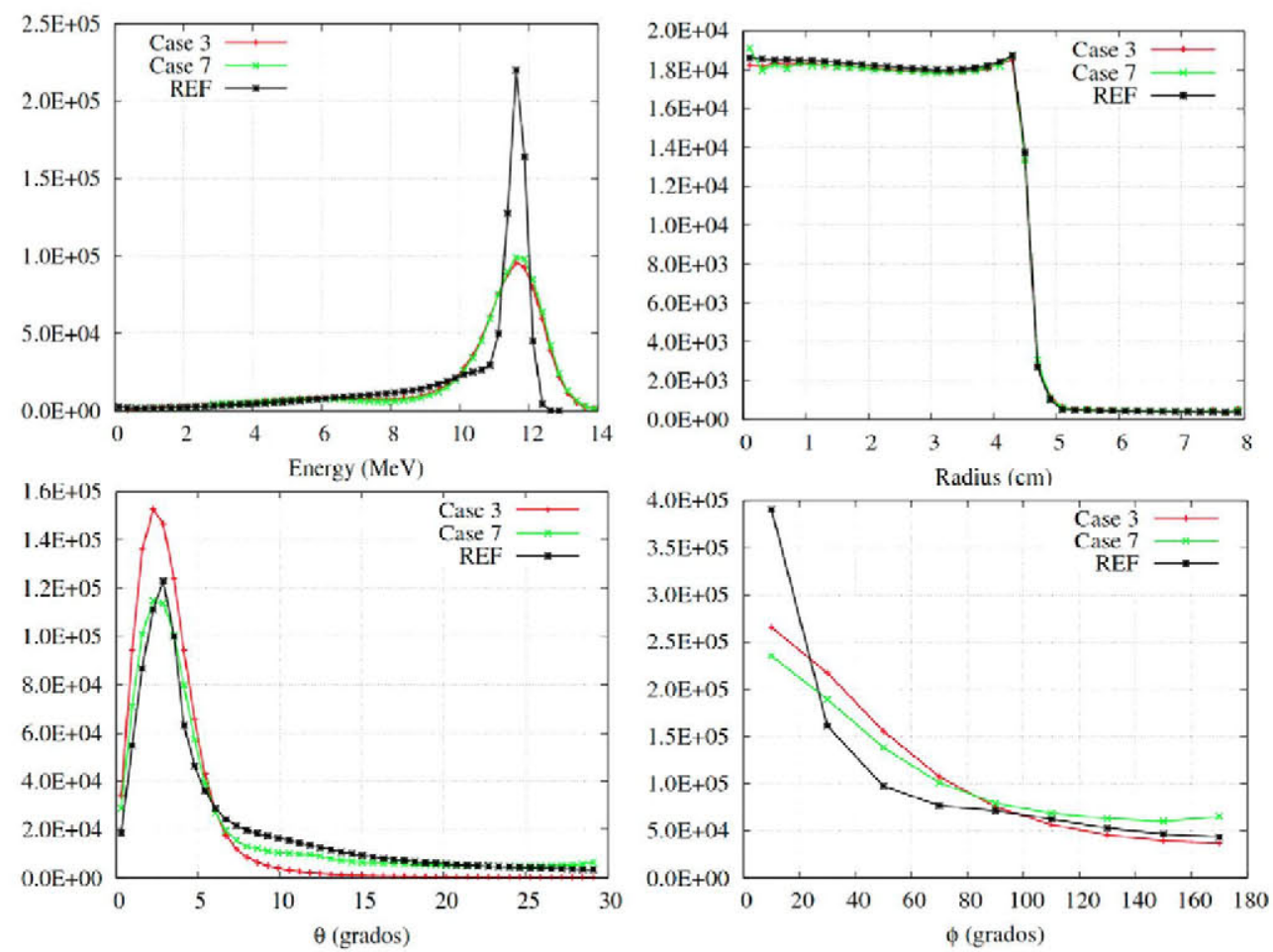

Figure 10. Distribution of electrons particles for the solution PHSP obtained with the case 3 and 7, and for $12 \mathrm{MeV}$ reference PHSP and $60 \mathrm{~cm}$ applicator length. The histograms are normalized to the same total number of particles.

these differences in the distribution on the angle $\phi$ have a limited impact on the dose which explains why the reconstruction of this distribution from dose measurements is difficult. This, together with its smooth distribution, motivated our use of a small number of bins for this variable, respect to the other ones. We want to note that the main reason why the $\theta$ angle affects the dose distribution significantly more than the $\phi$ angle, is because it is mainly responsible for how the electron beam is widened while penetrating the phantom, creating what it is called the penumbra in the dose (Attix 1986). A bad reproduction of the tails in the $\theta$ distribution yields large differences in the dose in the penumbra region.

The fact that angular distributions are not completely determined by the dose measurements employed in the fits for the cases 1 to 7 means that for the range in $\rho$ and $z$ considered, the doses do not depend as much on the angle distribution as on other properties of the PHSP, such as radial and energy distribution. If better agreement in these variables is needed, then a larger $z$ span for the measurements in air to be fed into the fitting procedure could be used.

\subsection{Dose comparison}

We show in tables 2 and 3 the result of the comparison of dose given by the solution PHSP to the one of the reference PHSP in terms of gamma function for the case of the electron source of $6 \mathrm{MeV}$ and 60 and $100 \mathrm{~cm}$ applicator lengths. The results obtained for the electron source of $12 \mathrm{MeV}$, both for 60 and $100 \mathrm{~cm}$ applicator length, are shown in table 4 . Results for 
Table 2. Test setups for which the gamma comparison of the dose produced by the solution PHSP to the one of the reference PHSP fulfills the $1 \%-1 \mathrm{~mm}$ critera (1), $2 \%-2 \mathrm{~mm}(2)$ or $3 \%-3 \mathrm{~mm}$ (3). If for a setup not even the $3 \%-3 \mathrm{~mm}$ criteria is fulfilled at least by $95 \%$ of the voxels, then the percentage of voxels with gamma less than 1 for the $3 \%-3 \mathrm{~mm}$ criteria is shown. In this table results for the $6 \mathrm{MeV}$ energy source and $60 \mathrm{~cm}$ applicator length, are shown.

\begin{tabular}{llllllll}
\hline & \multicolumn{3}{c}{ Water + air } & & \multicolumn{4}{c}{ Water only } \\
\cline { 2 - 3 } & Case 1 & Case 2 & & Case 3 & Case 4 & Case 5 & Case 6 \\
\hline Water & $(1)$ & $(1)$ & & $(1)$ & $(2)$ & $(3)$ & $(3)$ \\
Air & $(2)$ & $(2)$ & & 70.5 & 24.9 & 43.7 & 16.5 \\
Applicator & $(3)$ & $(3)$ & & 80.3 & 52.6 & 72.5 & 40.9 \\
Bevel & $(3)$ & $(2)$ & & 90.1 & 74.2 & 75.6 & 65.0 \\
Lung & $(1)$ & $(1)$ & $(2)$ & 90.1 & $(3)$ & 89.2 \\
Step & $(2)$ & $(2)$ & $(2)$ & $(2)$ & 92.9 & 90.8 \\
Lead & $(1)$ & $(1)$ & $(1)$ & $(2)$ & $(2)$ & $(3)$ \\
Bone & $(1)$ & $(1)$ & $(2)$ & $(2)$ & $(3)$ & 94 \\
\hline
\end{tabular}

Table 3. Same as table (2) for the $6 \mathrm{MeV}$ energy source and $100 \mathrm{~cm}$ applicator length.

\begin{tabular}{llllllll}
\hline & \multicolumn{3}{c}{ Water + air } & & \multicolumn{4}{c}{ Water only } \\
\cline { 2 - 3 } & Case 1 & Case 2 & & Case 3 & Case 4 & Case 5 & Case 6 \\
\hline Water & $(1)$ & $(1)$ & & $(1)$ & $(2)$ & $(2)$ & $(3)$ \\
Air & $(2)$ & $(2)$ & & 76.4 & 28.9 & 73.5 & 21.6 \\
Applicator & $(3)$ & $(3)$ & & 88.4 & 53.0 & 81.3 & 42.7 \\
Bevel & $(2)$ & $(2)$ & & $(3)$ & 72.3 & 93.7 & 64.4 \\
Lung & $(1)$ & $(1)$ & & $(2)$ & $(2)$ & $(2)$ & $(3)$ \\
Step & $(2)$ & $(2)$ & & $(2)$ & $(2)$ & $(2)$ & 93.0 \\
Lead & $(1)$ & $(1)$ & $(1)$ & $(2)$ & $(2)$ & $(3)$ \\
Bone & $(1)$ & $(1)$ & $(2)$ & $(2)$ & $(2)$ & $(3)$ \\
\hline
\end{tabular}

Table 4. Same as tables 2 and 3 for the $12 \mathrm{MeV}$ energy source.

\begin{tabular}{|c|c|c|c|c|}
\hline & \multicolumn{2}{|c|}{$\mathrm{SDD}=60 \mathrm{~cm}$} & \multicolumn{2}{|c|}{$\mathrm{SSD}=100 \mathrm{~cm}$} \\
\hline & Water only & Water + air & Water only & Water + air \\
\hline & Case 3 & Case 7 & Case 3 & Case 7 \\
\hline Water & (1) & (1) & (1) & (1) \\
\hline Air & 68.6 & (2) & 74.2 & (2) \\
\hline Applicator & 84.6 & (3) & 88.1 & (3) \\
\hline Bevel & (3) & (2) & (3) & (2) \\
\hline Lung & (2) & (2) & (2) & (2) \\
\hline Step & (2) & (2) & (2) & (1) \\
\hline Lead & (1) & (1) & (2) & (1) \\
\hline Bone & (2) & (1) & (2) & (1) \\
\hline
\end{tabular}

homogeneous objects of air and water and for the additional validation setups introduced in the precedent section are shown. In figures 11 and 12 dose profiles for the 'applicator', 'bevel', 'lung' and 'lead' setups are compared. 
(a) Applicator
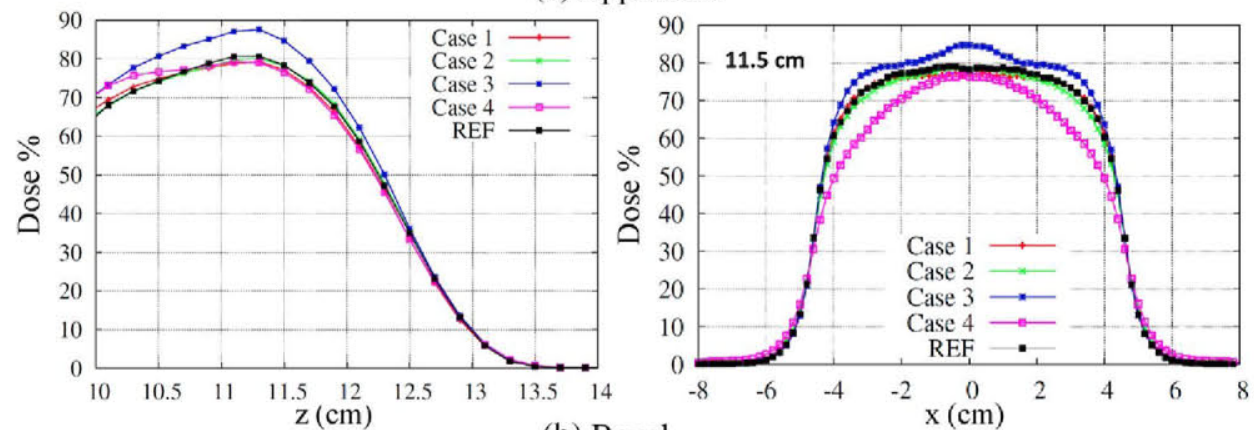

(b) Bevel
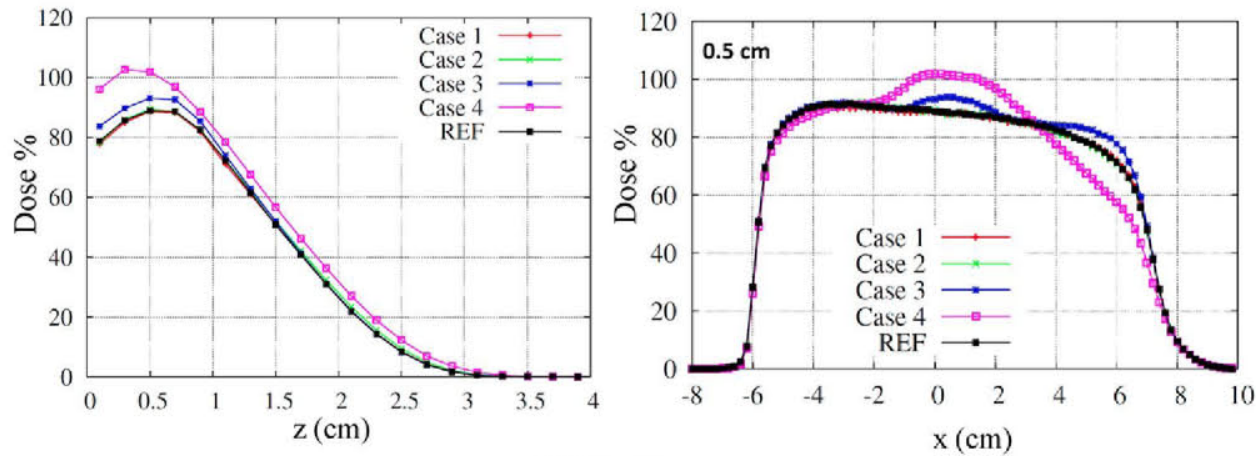

(c) Lung
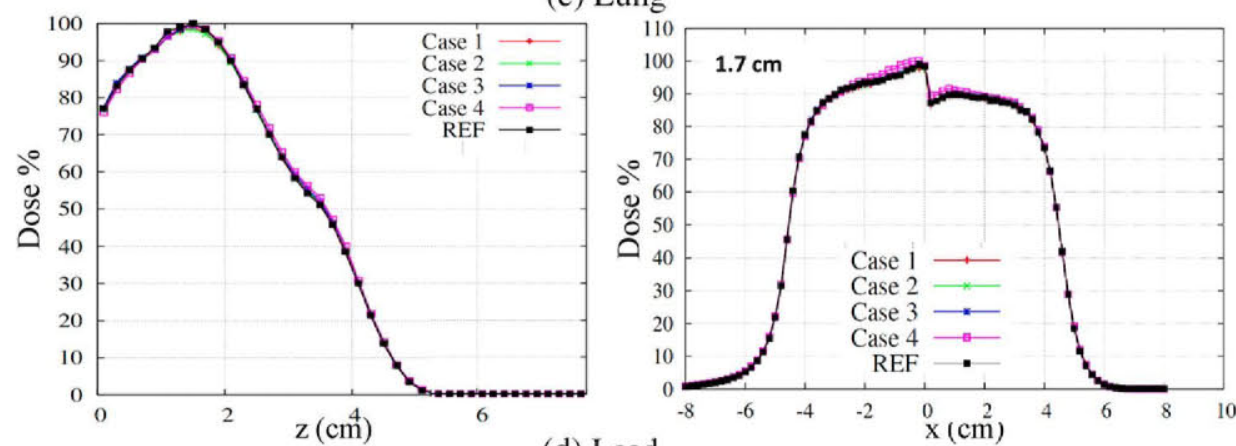

(d) Lead
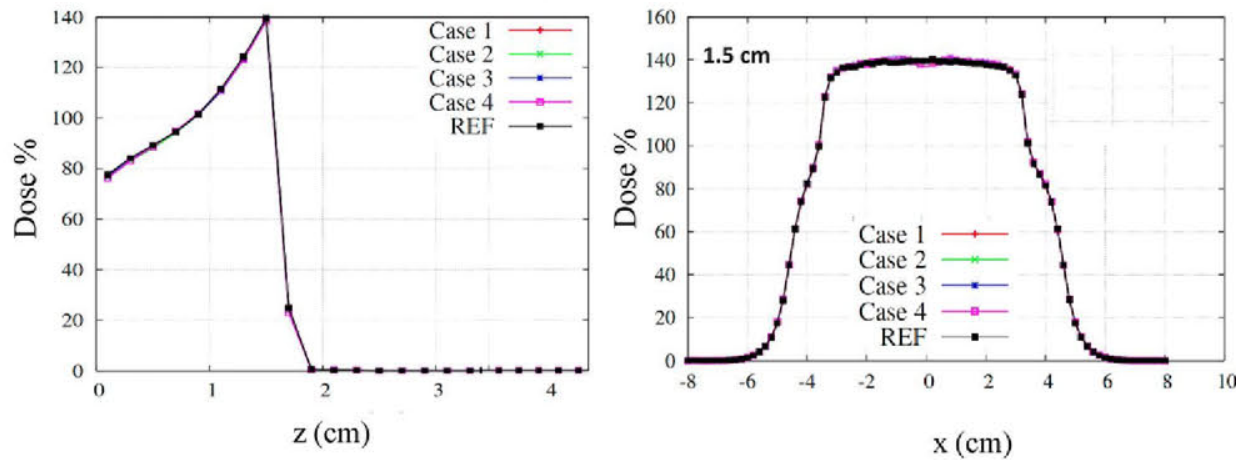

Figure 11. PDD and transverse dose profile near the maximum of the PDD for the 'applicator' $(a)$, 'bevel' $(b)$, 'lung' $(c)$ and 'lead' $(d)$ setups for $6 \mathrm{MeV}$ energy and $100 \mathrm{~cm}$ applicator length. The doses produced by solution and reference PHSP are compared. 
(a) Applicator
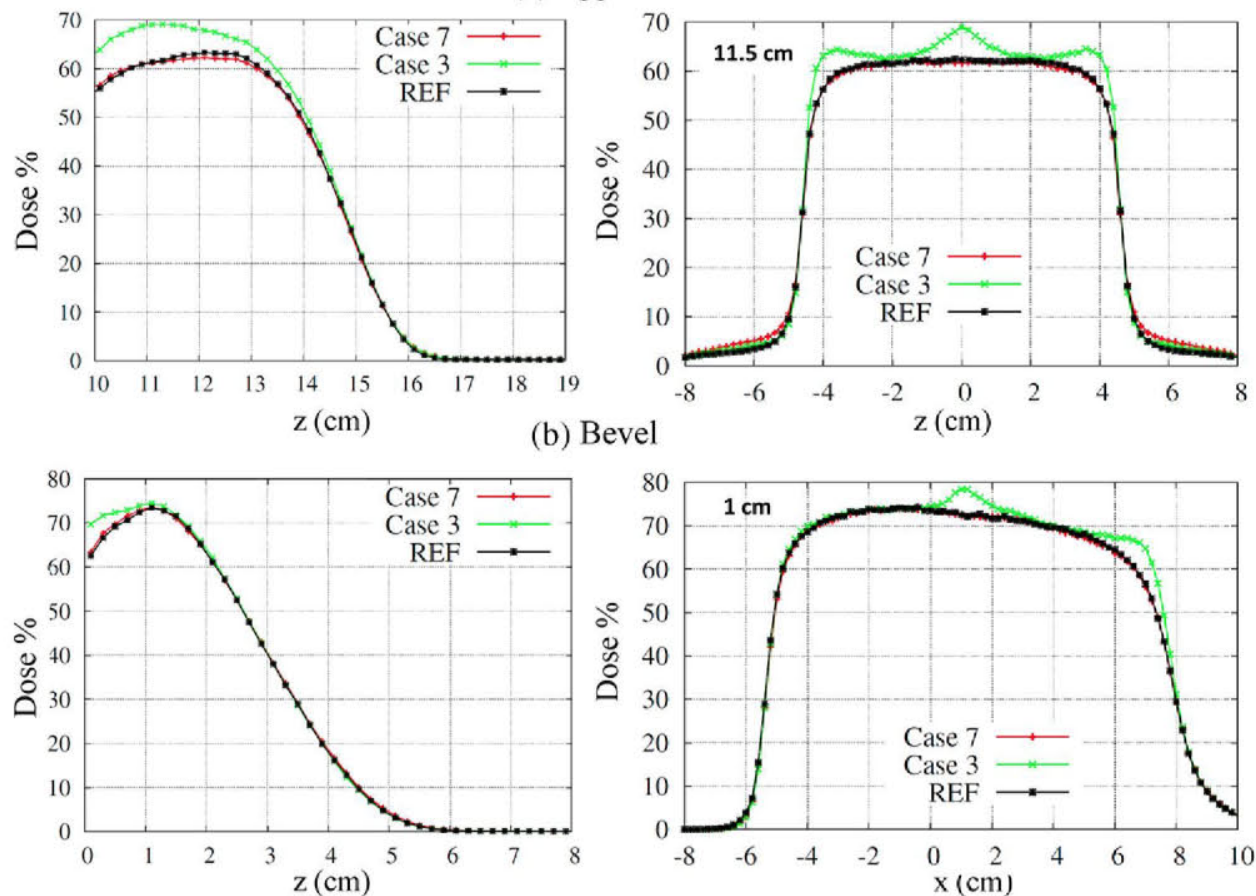

(c) Lung
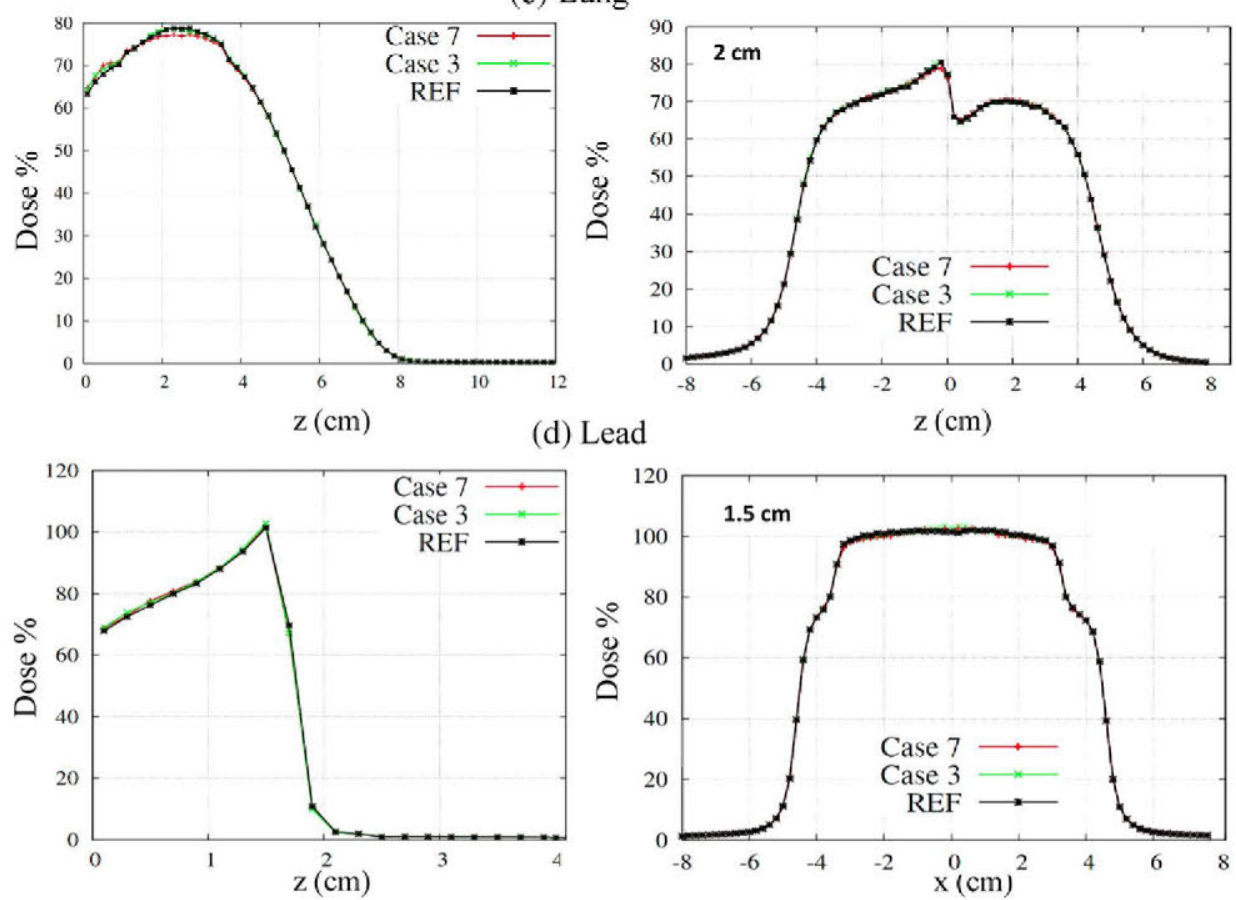

Figure 12. PDD and transverse dose profile near the maximum of the PDD for the 'applicator' $(a)$, 'bevel' $(b)$, 'lung' $(c)$ and 'lead' $(d)$ setups for the $12 \mathrm{MeV}$ cases and $60 \mathrm{~cm}$ applicator length. The doses produced by solution and reference PHSP are compared. 
Table 2 shows that both the solution PHSP of case 1 and case 2 fulfill the $3 \%-3 \mathrm{~mm}$ criteria in all tests. This is sufficient precision for IOERT treatments (Low et al 2003). And for the setups that do not involve air, they both pass the $1 \%-1 \mathrm{~mm}$ test. This shows that when both air and water data are employed in the fit, good results are obtained independently on the initial distributions of the angular variables employed. For cases 3 and 4, where only data in water are employed, when the initial flat angular dependence is employed, the solution PHSP yields dose away from the reference one. Table 3 shows similar results for the longer applicator. A similar conclusion can be extracted for the $12 \mathrm{MeV}$ energy source (table 4). Case 7, which uses both air and water data, fulfills at least the $3 \%-3 \mathrm{~mm}$ criteria for all the setups. As in the previous cases, case 3 produces doses departing more from the dose produced by the reference PHSP.

In figure 11 (6MeV energy source and $100 \mathrm{~cm}$ applicator) and 12 (12 MeV energy source and $60 \mathrm{~cm}$ applicator) some dose profiles are shown, where the agreement or disagreement indicated by the gamma test can be visualized. Notice that the dose produced with the solution PHSP for cases 1 and 2 (that is, including both air and water dose measurements in the fit) are nearly identical to the ones of the reference PHSP. On the other hand, the differences between the dose produced with solution PHSP reconstructed from only data in water, and the reference dose are very significant for the additional applicator length and beveled applicator end tests. This is expected, as these solution PHSPs did not use information of dose in air and they do not predict well dose in air, which plays a significant role in both applicator and beveled tests.

The conclusion is that if in the procedure only data in a water object is available to perform the fit, then one should try to use a realistic initial guess for the angular distribution, taken for instance from analytical expressions. Indeed, one of the outcomes of the comparison is that, besides the comparisons of the dose in air, very sensitive to small variations on the angular distributions, the most difficult test to pass for the solution PHSP is the extended applicator. This is also one of the most interesting outcomes of the MC PHSP, as having predictive power in this aspect would save a lot of measurements at IOERT services. The results of cases 5 and 6 reflect that, when only the energy is optimized to data in water (a common approach when fitting PHSP to data (Mihailescu et al 2006), if the angular distributions are not reasonable (as is the case with the ones obtained from flat starting guesses) unacceptable results are obtained On the other hand, case 5 shows that with sensible values for the initial angular and radial distributions, a working solution PHSP can be obtained employing data only in water and fitting only the energy distribution. We have also performed consecutive optimizations on the four variables, instead of optimizing the four of them at the same time. The results were worse, thus here we presented only results with the full optimization.

With regard to the application to real systems, the performance displayed by the procedure presented in this work indicates that one should worry more about the accuracy that can be achieved in the experimental measurements than about our ability to solve the inverse problem of finding the PHSP from dose. The accuracy of experimental measurements employed in the fit becomes an essential ingredient of the method, in order not to spoil its potential accuracy. We can say that with the method introduced in this work, one trades the difficulty of getting an accurate and precise MC model of the accelerator and applicator by the problem of obtaining accurate dose measurements in simple homogeneous media.

\section{Conclusions}

The method proposed for the determination of the PHSP of electron beams is suitable to obtain the PHSP for IOERT accelerators, with a modest effort from the IOERT services in providing 
dose measurements in homogeneous volumes of air and of water. Once the pre-computation of the binned sources is accomplished, which is a one-shot calculation that does not need to be repeated for different accelerators, the reconstructed PHSP is obtained in a short computing time (less than five minutes in the computer described previously).

Differences between the distributions of the reconstructed PHSP point out the non-uniqueness of the solution of this ill-posed problem. We have used several techniques to handle this. For instance, the space of solutions can be reduced by increasing the number of different measurements employed during the fit, like dose measurements in water and in air. Also, the use of a realistic initial PHSP in the iterative reconstruction has also been shown to help obtain better results (see how case 3 obtained better results than case 4 in tables 3 and 4).

We have verified that the reconstructed PHSP, although being slightly different than the actual PHSP distribution, leads to dosis profiles which are almost indistinguishable from the reference one shown (figures $11(c)$ and $(d)$ and figures $12(c)$ and $(d)$ ). We also evaluated how possible noise in the data may propagate into the PHSP solution. In this study, the noise was introduced by simulating the reference 'measurements' with fewer number of histories. The method has been shown to be robust and converge properly to a good working solution even in the presence of moderate noise in the data (see figure 7).

Dose distributions obtained from the solution PHSP for validation phantoms representative of typical IOERT scenarios, are in good agreement with the ones obtained from the reference PHSP, if the solution PHSP is obtained from data in air and water.

In view of these results, an ample program of determination of solution PHSPs against complete sets of real measurements (with different materials, applicators and systems) is currently being carried out in clinical IOERT settings at four major hospitals in Spain.

\section{Acknowledgments}

This work is supported by Comunidad de Madrid (ARTEMIS S2009/DPI-1802), Spanish Ministry of Science and Innovation (grants TEC2010-21619-C04, FPA2010-17142 and ENTEPRASE, PSE-300000-2009-5 and PRECISION grant IPT-300000-2010-3 and IPT2012-0401-300000), by European Regional Funds, by CDTI under the CENIT Programme (AMIT Project) and by CPAN, CSPD-2007-00042@Ingenio2010. Part of the calculations of this work were performed in the 'Clúster de Cálculo de Alta Capacidad para Técnicas Físicas' funded in part by UCM and in part by UE under FEDER programme.

\section{References}

Alber M et al 2008 Guidelines for the Verification of IMRT, Booklet No 9 ESTRO Brussels, Belgium Antolak J A, Bieda M R and Hogstrom K R 2002 Using Monte Carlo method to commission electron beams: a feasibility study Med. Phys. 29 771-86

Attix F H 1986 Introduction to Radiological Physics and Radiation Dosimetry (New York: Wiley)

Bakai A, Alber M and Nüsslin F 2003 A revision of the gamma evaluation concept for the comparison of dose distributions Phys. Med. Biol. 48 3543-53

Bakushinsky A B, Kokurin M Yu and Smirnova A 2011 Iterative Methods for Ill-posed Problems: an Introduction (Berlin: Walter de Gruyter $\mathrm{GmbH} \& \mathrm{Co} . \mathrm{KG}$ )

Beddar A S, Biggs P J, Chang S, Ezzell G A, Faddegon B A, Hensley F W and Mills M D 2006 Intraoperative radiation therapy using mobile electron linear accelerators: report of AAPM Radiation Therapy Committee Task Group No 72 Med. Phys. 33 1476-89

Björk P, Knöös T, Nilsson P and Larsson K 2000 Design and dosimetry characteristics of a soft-docking system for intraoperative radiation therapy Int. J. Rad. Oncol. Biol. Phys. 47 527-33 
Björk P, Nilsson P and Knöös T 2002a Dosimetry characteristics of degraded electron beams investigated by Monte Carlo calculations in a setup for intraoperative radiation therapy Phys. Med. Biol. 47 239-56

Björk P, Knöös T and Nilsson P 2002b Influence of initial electron beam characteristics on Monte Carlo calculated absorbed dose distributions for linear accelerator electron beams Phys. Med. Biol. $474019-41$

Bush K, Popescu I a and Zavgorodni S 2008 A technique for generating phase-space-based Monte Carlo beamlets in radiotherapy applications Phys. Med. Biol. 53 N337-47

Capote R, Jeraj R, Ma C-M, Rogers D W O, Sánchez-Doblado F, Sempau J, Seuntjens J and Siebers J V 2006 Phase-Space Database for External Beam Radiotherapy Summary Report of a Consultants' Meeting Technical Report INDC(NDS)-0484 (Vienna: International Nuclear Data Committee, INDC, IAEA)

Catalano M, Agosteo S, Moretti R and Andreoli S 2007 Monte Carlo simulation code in optimisation of the Intraoperative Radiation Therapy treatment with mobile dedicated accelerator J. Phys. Conf. Ser. $\mathbf{7 4} 021002$

Chetty I J et al 2007 Issues associated with clinical implementation of Monte Carlo-based photon and electron external beam treatment planning Report of the AAPM Task Group No 105 Med. Phys. 344818

Chvetsov A V and Sandison G A 2002 Reconstruction of electron spectra using singular component decomposition Med. Phys. 29 578-91

Deng J, Jiang S B, Pawlicki T, Li J and Ma C M 2001 Derivation of electron and photon energy spectra from electron beam central axis depth dose curves Phys. Med. Biol. 46 1429-49

Díaz-González JA, Calvo FA, Cortés J, García-Sabrido JL, Gómez-Espí M, Del Valle E, Muñoz-Jiménez F and Alvarez E 2006 Prognostic factors for disease-free survival in patients with $\mathrm{T} 3-4$ or $\mathrm{Np}$ rectal cancer treated with preoperative chemoradiation therapy, surgery, and intraoperative irradiation Int. J. Rad. Oncol. Biol. Phys. $641122-8$

Faddegon B, Balogh J, Mackenzie R and Scora D 1998 Clinical considerations of Monte Carlo for electron radiotherapy treatment planning Rad. Phys. Chem. 53 217-27

Faddegon B A and Blevis I 2000 Electron spectra derived from depth dose distribution Med. Phys. $27514-26$

Faddegon B, Schreiber E and Ding X 2005 Monte Carlo simulation of large electron fields Phys. Med. Biol. $50741-53$

Faddegon B A, Kawrakow I, Kubyshin Y, Perl J, Sempau J and Urban L 2009 The accuracy of EGSnrc, Geant4 and PENELOPE Monte Carlo systems for the simulation of electron scatter in external beam radiotherapy Phys. Med. Biol. 54 6151-63

Green P J 1990 Bayesian reconstructions from emission tomography data using a modified EM algorithm IEEE Trans. Med. Imag. 9 84-93

Guerra P, Udías J M, Herranz E, Valdivieso M, Calama J A, Illana C, Ledesma M and Santos A 2012 Optimization of Monte Carlo code for clinical simulation of electron beams Int. J. Radiat. Oncol. Biol. Phys. 84 (Suppl.) S870-1

Guerra P, González W, Ledesma-Carbayo M J, Cal-González J, Herranz E, Udías J M, Lallena A and Santos A 2010 Monte Carlo based dose estimation in intraoperative radiotherapy Nuclear Science Symp. Conf. Record NSS '10 IEEE (Knoxville, TN, USA) pp 3069-71

Guerra P et al 2014 Feasibility assessment of the interactive use of a Monte Carlo algorithm in treatment planning for intraoperative electron radiation therapy Phys. Med. Biol. 59 7159-79

Herraiz J L, España S, Vaquero J J, Desco M and Udías J M 2006 FIRST: fast iterative reconstruction software for (PET) tomography Phys. Med. Biol. 51 4547-65

Herranz E, Herraiz J L, Cal-González J, Corzo P M G, Guerra P and Udias J M 2011 Iterative reconstruction of whole accelerator phase spaces for intraoperative radiation therapy (IORT) from measured dose data Nucl. Sci. Symp. Conf. Rec. NSS '11 IEEE pp 2644-6

Herranz E, Herraiz J L, Guerra P, Cal-González J, Perez-Liva M, Rodriguez R, Illana C, Ledesm M J, Calama J A and Udias J M 2012a Iterative determination of clinical beam phase space from dose measurements Int. J. Radiat. Oncol. Biol. Phys. 84 S869

Herranz E et al 2012b Iterative reconstruction of clinical electron beam phase space for intra-operative radiation therapy from measured dose data 7 th Int. Conf. Int. Society for Intraoperative Radiation Therapy (ISIORT) (Baveno, Italy, 2012)

Hogstrom K R and Almond P R 2006 Review of electron beam therapy physics Phys. Med. Biol. $51455-89$ 
Iaccarino G, Strigari L, D'Andrea M, Bellesi L, Felici G, Ciccotelli A, Benassi M and Soriani A 2011 Monte Carlo simulation of electron beams generated by a $12 \mathrm{MeV}$ dedicated mobile IOERT accelerator Phys. Med. Biol. 56 4579-96

Ibañez García P 2012 Validación de modelos de aceleradores para Radioterapia Intraoperatoria Experimentos y simulaciones Master Thesis Universidad Complutense de Madrid

ICRU Report 441989 Tissue substitutes in Radiation Dosimetry and Measurement (Bethesda, MD: International Commission on Radiation Units and Measurements) (www.i cru.org/home/reports/ tissue-substitutes-in-radiation-dosimetry-and-measurement-report-44)

Ishkhanov B S, Pakhomov N I, Shvedunov N V, Shvedunov V I, Msu S, Gorbachev V P and Ssu R 2004 Conceptual design of the miniature electron accelerator dedicated to IOERT Proc. RuPAC XIX (Dubna, Russia) pp 474-6

Jabbari N and Hashemi-malayeri B 2009 Monte Carlo modeling of electron beams from a NEPTUN $10 \mathrm{PC}$ medical linear accelerator Nukleonika $\mathbf{5 4} 233-8$

Janssen J J, Korevaar E W, van Battum L J, Storchi P R and Huizenga H 2001 A model to determine the initial phase space of a clinical electron beam from measured beam data Phys. Med. Biol. $46269-86$

Janssen R W J, Faddegon B a and Dries W J F 2008 Prototyping a large field size IOERT applicator for a mobile linear accelerator Phys. Med. Biol. 53 2089-102

Kusters M et al 2010 Results of European pooled analysis of IOERT-containing multimodality treatment for locally advanced rectal cancer: adjuvant chemotherapy prevents local recurrence rather than distant metastases Annal. Oncol. 21 1279-84

Lamanna E, Gallo A, Russo F, Brancaccio R, Soriani A and Strigari L 2012 Intra-operative radiotherapy with electron beam Modern Practices in Radiation Therapy ed G Natanasabapathi (Rijeka: InTech)

Low D A and Dempsey J F 2003 Evaluation of the gamma dose distribution comparison method Med. Phys. 30 2455-64

Ma C-M and Jiang Steve B 1999 Monte Carlo modelling of electron beams from medical accelerators Monte Carlo modelling of electron beams from medical accelerators Phys. Med. Biol. 44 157-89

Ma C M et al 1999 Clinical implementation of a Monte Carlo treatment planning system Med. Phys. $262133-43$

McDermott P N, He T and DeYoung A 2003 Dose calculation accuracy of lung planning with a commercial IMRT treatment planning system J. Appl. Clin. Med. Phys. 4 341-51

Mihailescu D, Pimpinella M, Guerra A S and Laitano R F 2006 Comparison of measured and Monte Carlo calculated dose distributions the novac7 (B) linear accelerator Rom. J. Phys. 51 729-39

Nevelsky A, Bernstein Z, Bar-Deroma R, Kuten A and Orion I 2010 Design and dosimetry characteristics of a commercial applicator system for intra-operative electron beam therapy utilizing ELEKTA Precise accelerator J. Appl. Clin. Med. Phys./American College of Medical Physics 113244

Pascau J, Santos J A, González G, Ferrer F, Ramos A, Meiriño R, Calvo F and Desco M 2011 Virtual planning for IEORT: radiance main features and recent improvements GEC-ESTRO ISIOERT Europe Conf. (Londres, Reino Unido)

Pascau J, Santos Miranda J A, Calvo F A, Bouché A, Morillo V, González-San Segundo C, Ferrer C, López Tarjuelo J and Manuel D 2012 An innovative tool for intraoperative electron beam radiotherapy simulation and planning: description and initial evaluation by radiation oncologists Inter. J. Radiat. Oncol. Biol. Phys. 83 e287-95

Perez Liva M 2012 Simulación de aceleradores para Radioterapia Intraoperatoria Master Thesis Universidad Complutense de Madrid

Picardi L, Ronsivalle C, Tata A, Vignati A and Rome C R F F 2000 The Italian IORT Project Proc. EPAC (Vienna, Austria) pp 2545-7

Popescu I and Bush K 2005 Commissioning of virtual linacs for Monte Carlo simulations by optimizing photon source characteristics Radiother. Oncol. 76 (Suppl. 2) S43

Righi S, Karaj E, Giuseppe F and Di M F 2013 Dosimetric characteristics of electron beams produced by two mobile accelerators, Novac7 and Liac, for intraoperative radiation therapy through Monte Carlo simulation J. Appl. Clin. Med. Phys./American College of Medical Physics 143678

Rodriguez M, Sempau J and Brualla L 2013 PRIMO: a graphical environment for the Monte Carlo simulation of Varian and Elekta linacs Strahlenther. Onkol. 189 881-6

Rogers D W 2006 Fifty years of Monte Carlo simulations for medical physics Phys. Med. Biol. $51287-301$ 
Rogers D W O, Faddegon B A, Ding G X, Ma C M and We J 1995 BEAM: a Monte Carlo code to simulate radiotherapy treatment units $\mathrm{Med}$. Phys. 22 507-24

Russo G, Casarino C, Arnetta G, Candiano G, Stefano A, Alongi F, Borasi G, Messa C and Gilardi M C 2012 Dose distribution changes with shielding disc misalignments and wrong orientations in breast IOERT: a Monte Carlo-GEANT4 and experimental study J. Appl. Clin. Med. Phys./American College of Medical Physics 133817

Santos J A, Pascau J, Lardiés D, Desco M and Calvo F A 2011 Initial clinical experience of Pencil Beam dose modelling for intraoperative electron radiation therapy (IOERT) GEC-ESTRO ISIOERT Europe Conf. (Londres, Reino Unido)

Santos J A et al 2011 Intraoperative electron radiation therapy pre-planning using Radiance new features. The need for common protocols revisited GEC-ESTRO ISIOERT Europe Conf. (Londres, Reino Unido)

Sempau J and Andreo P 2006 Configuration of the electron transport algorithm of PENELOPE to simulate ion chambers Phys. Med. Biol. $513533-48$

Sempau J, Badal A and Brualla L 2011 A PENELOPE-based system for the automated Monte Carlo simulation of clinacs and voxelized geometries-application to far-from-axis fields Med. Phys. $385887-95$

Sempau J, Fernández-Varea J M, Acosta E and Salvat F 2003 Experimental benchmarks of the Monte Carlo code Penelope Nucl. Instrum. Methods B 207 107-23

Sempau J, Sánchez-Reyes A, Salvat F, ben Tahar H O, Jiang S B and Fernández-Varea J M 2001 Monte Carlo simulation of electron beams from an accelerator head using PENELOPE Phys. Med. Biol. $461163-86$

Sempau J, Wilferman S J and Bielajew A F 2000 DPM, a fast, accurate Monte Carlo code optimized for photon and electron radiotherapy treatment planning dose calculations Phys. Med. Biol. $452263-91$

Siebers J V, Keall P J, Nahum A E, Mohan R 2000 Converting absorbed dose to medium to absorbed dose to water for Monte Carlo based photon beam dose calculations Phys. Med. Biol. 45 983-95

Wysocka-rabin A, Adrich P and Wasilewski A 2011 Monte Carlo study of a new mobile electron accelerator head for intraoperative radiation therapy (IOERT) Prog. Nucl. Sci. Technol. 2 181-6

Yepes P, Randeniya S, Taddei P J and Newhauser W D 2009 A track-repeating algorithm for fast Monte Carlo dose calculations of proton radiotherapy Nucl. Technol. 168 736-40 